\title{
A Hybrid Peridynamics-SPH Simulation of Soil Fragmentation by Blast Loads of Buried Explosive
}

Houfu Fan, Guy Leshem Bergel, Shaofan Li*

Department of Civil and Environmental Engineering, University of California, Berkeley, CA94720

\begin{abstract}
In this work, we employ both the state-based peridynamics and the smoothed particle hydrodynamics (SPH) to simulate soil fragmentation/ejection induced by the blast of buried explosives. We use peridynamics representation to model soil medium, and we use SPH representation to model explosive gas. The key of the simulation is the coupling of the two. In the peridynamics-SPH computational domain, there is an interphase zone, in which a peridynamic particle can have SPH particles within its own horizon, while an SPH particle can have peridynamic particles within its supporting domain. The interactions of the peridynamic and SPH particles in this interphase zone are discussed. By assuming the equivalence of the two methods in the current configuration, we study how to choose simulation parameters that can seamlessly couple the two methods. A Drucker-Prager plasticity soil model at finite strain is used for soil medium and a charge model of equation of state is used to model the TNT explosive. Numerical examples are carried out to simulate soil fragmentation/ejection induced by the shock waves from the blastic loadings of the buried explosive. It is shown that the numerical results are in general agreement with that of the experiment.
\end{abstract}




\section{INTRODUCTION}

Modeling and simulation of soil fragmentation/ejection generated by shock waves from blast loading has been a challenge in computational mechanics research because of massive fragments that are almost impossible to use any interpolation field to represent. The particle method is almost the only method that can correctly represent the interaction of the soil and the explosive gas, and characterize the expansion of the explosive gas. Unfortunately for most particle methods, the ones that are accurate are computationally expensive, and the ones that are fast are inaccurate or lack of convergence.

In recent years, Silling [1] proposed a computational nonlocal continuum mechanics method, which he coined as peridynamics. It is not only an efficient particle method, but also a convergent particle method. Peridynamics has two distinct formulations: the bond based peridynamics and the state based peridynamics. The state-based peridynamics, in particular, is a meshfree particle method that not only can accommodate various macroscale constitutive relations, but also runs fast and is convergent. It has solved quite some material and structural failure problems in engineering mechanics applications, due to its capabilities in representing and capturing discontinuous deformation in solids and structures during their failure processes. The state-based peridynamics is a nonlocal continuum theory of solid mechanics that replaces conventional partial differential equations with displacement involved integral equations, which can characterize mechanical behaviors involving significant discontinuities, for instance, crack. One of the most distinguishing features of the state-based peridynamics is that it can seamlessly incorporate any constitutive relations in classical continuum mechanics framework. Thus in contrast with molecular dynamics [2], which can only handle problems with limited time or size scales, the corresponding peridynamics particle formulation provides a computationally efficient 
algorithm to simulate large deformations and fragmentation at macroscale. State-based peridynamics has been successfully applied to a lot of fracture or damage processes. For example, Warren et al. (2009) simulated the deformation and fracture of solid materials [3]; Foster et al. (2010) investigated the viscoplasticity a bar under impact [4] ; Weckenr and Mohamed (2013) implemented a state-based viscoelastic peridynamics model [5]; Tuniki (2012) employed state-based peridynamics to predict the fracture processes of concrete [6]; and Xin et al. (2015) simulated the fragmentation of geomaterial induced by impluse loads using peridynamics [7].

In the simulation of soil fragementation/ejection resulting from blast loading, the expansion of the explosive gas will deform and distort the local soil configuration. Thus, conventional mesh-based Lagrangian methods such as finite element method (FEM) will fail because of severe mesh distortion. In addition, it is almost impossible to trace the fastmoving and evolving interface front by using the conventional Eulerian mesh technique such as the finite volume method [8]. We would like to point out that the interaction between the fragmented soil medium and the explosive gas will make the shock wave fronts in the soil and in the gas becoming complex surfaces in three-dimensional space, which also poses serious challenge even for some advanced techniques such as level set method.

The SPH method has been utilized to simulate under water explosion [9]. But as is well known, the SPH method is developed to solve hydrodynamics problems, and is not so accurate when it comes to solid mechanics. In this work, a state-based peridynamics-SPH (PD-SPH) coupling scheme is employed to address the theoretical and numerical issues related to the fragmentation of soil under a high strain rate loading. On one hand, the state-based peridynamics offers advantages in handling discontinuities. On the other hand, the SPH methodology [10] can deal with the dynamic process of the explosive gas 
and the moving boundary, given that it is a Lagrangian particle method with an Eulerian kernel function [11].

To accurately characterize soil fragmentation driven by blast loads in the proposed hybrid PD-SPH framework, one needs the correct constitutive relations for both the soil and the explosives. For the explosive part, the one used in [9] is adopted. Soils usually present great nonlinearities after plastic yielding, with pressure-sensitive yield surfaces [12]. In the literature, there are many successful constitutive models for soils, such as the Drucker-Prager (DP) model [13], the Mohr-Coulomb (MC) model [12] and the MatsuokaNakai (MN) model [14], and etc. In this work, the Drucker-Prager (DP) plasticity model is adopted to characterize the plastic behaviors of the soil. The DP model has been extensively used in numerical simulations of soil, rock and concrete structures [15]. It reduces to a familiar $J_{2}$ plasticity form when the pressure sensitivity part vanishes. The material parameters of the DP model for soils are well documented with experimental calibrations [16]. To conform with the large deformation and distortion, the DP model at finite deformation with the Hughes and Winget algorithm is rephrased in the statebased peridynamics framework for the constitutive update. In [17], the preliminary study of the PD-SPH coupling approach has been reported by the present author. In this work, we aim to present a more detailed explanation on the equivalence of the two methods, especially in the coupling zone. In addition, the real time simulation and the corresponding experiment validation of under buried soil explosion are performed.

The paper is organized into six sections. In Section $\Pi$, we first outline the state-based peridynamics and SPH methods, with a standard Monagphan type artificial viscosity incorporated into the formulations of both methods, which can help preventing nonphysical particle interpenetrations. In Section III. we discuss the interaction of peridynamics and $\mathrm{SPH}$ particles in the interphase zone. By assuming the equivalence of two formulations in 
the current configuration, we propose a coupling algorithm between peridynamics and $\mathrm{SPH}$. In Section IV, a Drucker-Prager plasticity soil model is presented at finite strain, with the Hughes-Winget algorithm rephrased in state-based peridynamics framework for the constitutive update. In Section $\mathrm{V}$. Numerical examples are carried out to verify the soil modeling in the state-based peridynamics framework. Finally, by using the proposed PD-SPH coupling algorithm, we present the simulation of soil fragmentation/ejection induced by the blastic loadings of buried explosive. The comparison of the early soil deformations from an explosion experiment and the corresponding simulation results proves the validity of the proposed coupling scheme to certain extent. explosion experiment To close our presentation, we make a few remarks in Section VI.

\section{Overview of the State-Based Peridynamics ANd SPH}

In this section, we first briefly review the state-based peridynamics, in which we incorporate the artificial viscosity into the peridynamics formulation so as to prevent nonphysical particle interpenetration. Then we outline the basics of SPH method, including the SPH equation of state TNT explosive for simulating blast loading.

\section{State-based peridynamics}

State-based peridynamics is a nonlocal continuum theory of solid mechanics that replaces conventional partial differential equations with displacement involved integral equations [18]. In the state-based peridynamics, a continuum body $\Omega_{0}$ is considered to be a group of discretized material particles $\mathbf{X}_{A}$ with associated volume $V_{A}^{0}$ and mass density $\rho_{A}^{0}$, where $A=1,2, \ldots, \infty$ is the particle index. It is assumed that a material particle $\mathbf{X}_{A}$ only interacts with particles $\mathbf{X}_{B}$ within a local region, called as a horizion $\mathcal{H}_{\mathbf{X}^{A}}$. Typically, a horizon is chosen to be a circle (2D) or a sphere (in 3D), with the radius $\delta \mathbf{x}_{A}$ named as the horizon 
size of particle $\mathbf{X}_{A}$. All the particles $\mathbf{X}_{B}, B=1,2, \ldots, n_{A}$ inside the horizon $\mathcal{H}_{\mathbf{X}_{A}}$ are called as the family of particle $\mathbf{X}_{A}$ and $n_{A}$ denotes the total number of particles in this family. The relative position vector pointing from particle $\mathbf{X}_{A}$ to $\mathbf{X}_{B}$ in the reference configuration is called a bond, which shall be denoted as

$$
\mathbf{X}_{A \rightarrow B}:=\mathbf{X}_{B}-\mathbf{X}_{A}
$$

The horizon $\mathcal{H}_{\mathbf{x}_{A}}$ is defined by

$$
\mathcal{H}_{\mathbf{X}_{A}}:=\left\{\mathbf{X}_{A \rightarrow B} \in \mathcal{R}^{3}|0 \leq| \mathbf{X}_{A \rightarrow B} \mid \leq \delta_{\mathbf{X}_{A}}\right\}
$$

Under certain motion or deformation $\chi$, the continuum body $\Omega_{0}$ in the reference configuration deforms and evolves to $\Omega$ in the current configuration. Correspondingly, the material points $\boldsymbol{X}_{A}$ and $\boldsymbol{X}_{B}$ map to $\mathbf{x}_{A}$ and $\mathbf{x}_{B}$ respectively. The bond $\mathbf{X}_{A \rightarrow B}$ becomes

$$
\mathbf{x}_{A \rightarrow B}=\mathbf{x}_{B}-\mathbf{x}_{A}=\underline{\mathbf{Y}}\left(\mathbf{X}_{A \rightarrow B}\right)
$$

where $\underline{\mathbf{Y}}$ is a nonlinear quantity called the deformation state, which is a vector-state, i.e, a mathematical object in peridynamics theory in analogous to a tensor in classical continuum mechanics. Here, the deformation state $\underline{\mathbf{Y}}$ maps the undeformed bond $\mathbf{X}_{A \rightarrow B}$ to a deformed bond $\mathbf{x}_{A \rightarrow B}$.

Aside from the deformation state, another important vector-state in the peridynamic theory is the force state $\underline{\mathbf{T}}$, with which the balance of the linear momentum can be written as

$$
\rho_{A}^{0} \ddot{\mathbf{u}}=\int_{\mathcal{H}_{\mathbf{X}_{A}}}\left[\underline{\mathbf{T}}\left(\mathbf{X}_{A}, \mathbf{X}_{A \rightarrow B}\right)-\underline{\mathbf{T}}\left(\mathbf{X}_{B}, \mathbf{X}_{B \rightarrow A}\right)\right] d V_{\mathbf{X}_{B}}+\rho_{A}^{0} \mathbf{b},
$$

where $\mathbf{u}$ is the displacement and $\mathbf{b}$ is the external body force.

By establishing an equivalence of the strain energy functionals [1, 18], one can obtain the relation between the force state and the first Piola-Kirchhoff stress tensor $\mathbf{P}$ of classical 
continuum mechanics as

$$
\underline{\mathbf{T}}\left(\mathbf{X}_{A}, \mathbf{X}_{A \rightarrow B}\right)=\omega\left(\left|\mathbf{X}_{A \rightarrow B}\right|\right) \mathbf{P}_{\mathbf{X}_{A}} \mathbf{K}_{\mathbf{X}_{A}}^{-1} \cdot \mathbf{X}_{A \rightarrow B}
$$

where $\omega\left(\left|\mathbf{X}_{A \rightarrow B}\right|\right)$ is a positive scalar influence function depending on the distance of the two particles, $\left|\mathbf{X}_{A \rightarrow B}\right|$, and $\mathbf{K}_{\mathbf{X}_{A}}$ is the reference shape tensor, defined as

$$
\begin{aligned}
\mathbf{K}_{\mathbf{x}_{A}} & =\int_{\mathcal{H}_{\mathbf{x}_{A}}} \omega\left(\left|\mathbf{X}_{A \rightarrow B}\right|\right) \mathbf{X}_{A \rightarrow B} \otimes \mathbf{X}_{A \rightarrow B} d V_{\mathbf{X}_{B}} \\
& =\sum_{\mathbf{X}_{B} \in \mathcal{H}_{\mathbf{x}_{A}}} \omega\left(\left|\mathbf{X}_{A \rightarrow B}\right|\right) \mathbf{X}_{A \rightarrow B} \otimes \mathbf{X}_{A \rightarrow B} V_{B}^{0}
\end{aligned}
$$

To compute the first Piola-Kirchhoff stress tensor $\mathbf{P}_{\mathbf{X}_{A}}$, one needs to first obtain the deformation gradient tensor $\mathbf{F}_{\mathbf{X}_{A}}$, which can then be substituted into the constitutive relations in classical continuum mechanics. For this purpose, the following shape tensor $\mathbf{N}_{\mathbf{X}_{A}}$ is introduced,

$$
\begin{aligned}
\mathbf{N}_{\mathbf{X}_{A}} & =\int_{\mathcal{H}_{\mathbf{x}_{A}}} \omega\left(\left|\mathbf{X}_{A \rightarrow B}\right|\right) \mathbf{x}_{A \rightarrow B} \otimes \mathbf{X}_{A \rightarrow B} d V_{\mathbf{X}_{B}} \\
& =\sum_{\mathbf{X}_{B} \in \mathcal{H}_{\mathbf{x}_{A}}} \omega\left(\left|\mathbf{X}_{A \rightarrow B}\right|\right) \mathbf{x}_{A \rightarrow B} \otimes \mathbf{X}_{A \rightarrow B} V_{B}^{0}
\end{aligned}
$$

If we assume that the deformations of all the bonds $\mathbf{X}_{A \rightarrow B}$ within a horizon $\mathcal{H}_{\mathbf{x}_{A}}$ is uniform, then the corresponding deformed bonds can be expressed as

$$
\mathbf{x}_{A \rightarrow B}=\underline{\mathbf{Y}}\left(\mathbf{X}_{A \rightarrow B}\right)=\mathbf{F}_{\mathbf{X}_{A}} \cdot \mathbf{X}_{A \rightarrow B}
$$

where $\mathbf{F}_{\mathbf{X}_{A}}$ is a second-order tensor and can be viewed as the approximated deformation gradient at particle $A$. Substitute Eq. (8) into (7) and combine with Eq. (6), one may get

$$
\mathbf{N}_{\mathbf{X}_{A}}=\mathbf{F}_{\mathbf{X}_{A}} \cdot\left[\sum_{\mathbf{X}_{B} \in \mathcal{H}_{\mathbf{x}_{A}}} \omega\left(\left|\mathbf{X}_{A \rightarrow B}\right|\right) \mathbf{X}_{A \rightarrow B} \otimes \mathbf{X}_{A \rightarrow B} V_{B}^{0}\right]=\mathbf{F}_{\mathbf{X}_{A}} \cdot \mathbf{K}_{\mathbf{X}_{A}}
$$

Notice that the shape tensor $\mathbf{K}_{\mathbf{X}_{A}}$ is calculated based on the reference configuration. As long as there are enough bonds within the horizon $\mathcal{H}_{\mathbf{X}_{A}}$, matrix $\mathbf{K}_{\mathbf{X}^{A}}$ is invertible. Therefore, the approximated deformation gradient can be obtained as

$$
\mathbf{F}_{\mathbf{X}_{A}}=\mathbf{N}_{\mathbf{X}_{A}} \cdot \mathbf{K}_{\mathbf{X}_{A}}^{-1}
$$


Although the state-based peridynamics is very promising, it has been observed in numerical simulations that certain nonphysical deformation modes may happen, which can lead to particle inter-penetrations [19, 20]. This issue is extremely prominent for large deformation problems that have high strain rates resulting from explosive load. In this work, we propose to introduce an artificial viscous stress term into the original peridynamics formulation. In specific, the following Monaghan type artificial viscosity used by [9] is considered,

$$
\Pi_{A B}= \begin{cases}\frac{-\alpha \bar{c}_{A B} \phi_{A B}+\beta \phi_{A B}^{2}}{\bar{\rho}_{A B}}, & \mathbf{v}_{A B} \cdot \mathbf{x}_{A B} \leq 0 \\ 0, & \mathbf{v}_{A B} \cdot \mathbf{x}_{A B}>0\end{cases}
$$

where

$$
\begin{aligned}
\phi_{A B} & =\frac{\bar{\delta}_{A B} \mathbf{v}_{A B} \cdot \mathbf{x}_{A B}}{\left|\mathbf{x}_{A B}\right|^{2}+\left(\varphi \bar{\delta}_{A B}\right)^{2}} \\
\bar{c}_{A B} & =\frac{1}{2}\left(c_{A}+c_{B}\right) \\
\bar{\rho}_{A B} & =\frac{1}{2}\left(\rho_{A}+\rho_{B}\right) \\
\bar{\delta}_{A B} & =\frac{1}{2}\left(\delta_{A}+\delta_{B}\right) \\
\mathbf{v}_{A B} & =\mathbf{x}_{B}-\mathbf{x}_{A} \\
\mathbf{x}_{A B} & =\mathbf{x}_{B}-\mathbf{x}_{A}
\end{aligned}
$$

In these equations above, $\alpha$ and $\beta$ are constant parameters and they are set to be 1.0. $\varphi$ is the variable used to prevent the overlapping of the two particles $A$ and $B$ and is set to be 0.1. $\bar{c}_{A B}, \bar{\rho}_{A B}$ and $\delta_{A B}$ are the average solid velocity, density and interacting distance, respectively. The following Cauchy stress corresponding to the artificial viscosity is introduced,

$$
\sigma^{\text {viscous }}:=\sum_{\mathbf{X}_{B} \in \mathcal{H}_{\mathbf{x}_{A}}} \omega\left(\mathbf{X}_{A \rightarrow B}\right) \Pi_{A B} \mathbf{I} V_{B}
$$


which can be converted into the first Piola-Kirchhoff viscous stress as

$$
\mathbf{P}^{\text {viscous }}=J \sigma_{\text {viscous }} \mathbf{F}^{-T}
$$

In the end, the modified force state with artificial viscosity can be written as

$$
\underline{\mathbf{T}}\left(\mathbf{X}_{A}, \mathbf{X}_{A \rightarrow B}\right)=\omega\left(\left|\mathbf{X}_{A \rightarrow B}\right|\right)\left(\mathbf{P}_{\mathbf{X}_{A}}-\mathbf{P}_{\mathbf{X}_{A}}^{v i s c o u s}\right) \mathbf{K}_{\mathbf{X}_{A}}^{-1} \cdot \mathbf{X}_{A \rightarrow B}
$$

\section{Basic of SPH and essential formulation for blast loading}

The SPH method was developed for hydrodynamics problems, which are usually expressed in the form of partial differential equations of density, velocity, energy and so forth. There are two key steps in the formulation of the SPH methodology. The first one is the kernel approximation and the second one is particle approximation [11].

In the first step, a continuous field $f(\mathbf{x})$ and its gradient $\nabla f(\mathbf{x})$ is approximated as

$$
\langle f(\mathbf{x})\rangle=\int_{\Omega} f(\mathbf{y}) W(|\mathbf{x}-\mathbf{y}|) d V_{\mathbf{y}}
$$

where \langle\rangle is the kernel approximation operator [21], $W$ is the so-called kernel function. Using the smoothing function, the gradient of $f(\mathbf{x})$ can be written as

$$
\langle\nabla f(\mathbf{x})\rangle=-\int_{\Omega} f(\mathbf{y}) \nabla_{\mathbf{x}} W(|\mathbf{x}-\mathbf{y}|) d V_{\mathbf{y}}
$$

where the minus sign results from the integration by parts, which is standard in SPH [22]. The particle approximation basically invovles the way to represent the target continuum into a finite number of particles that carry individual mass and volume. By doing this, any continuous integral representation can be directly converted into discretized summations. For instance, Eqs. (21) and (22) can be written in the corresponding discretized forms as

$$
\left\langle f\left(\mathbf{x}_{A}\right)\right\rangle=\sum_{\mathbf{x}_{B} \in \mathcal{S}_{\mathbf{x}_{A}}} f\left(\mathbf{x}_{B}\right) W\left(\left|\mathbf{x}_{A}-\mathbf{x}_{B}\right|\right) V_{B}
$$


and

$$
\left\langle\nabla f\left(\mathbf{x}_{A}\right)\right\rangle=-\sum_{\mathbf{x}_{B} \in \mathcal{S}_{\mathbf{x}_{A}}} f\left(\mathbf{x}_{B}\right) \nabla_{\mathbf{x}_{A}} W\left(\left|\mathbf{x}_{A}-\mathbf{x}_{B}\right|\right) V_{B}
$$

where $\mathcal{S}_{\mathbf{x}_{A}}$ represents the supporting (influence) domain of particle $\mathbf{x}_{A}$, similar to the definition of horizon $\mathcal{H}_{\mathbf{x}_{A}}$ in the state-based peridynamics. In Eq. (24), by considering a constant field $f(\mathbf{x})=1$ and a linear field $f(\mathbf{x})=\mathbf{x}$, one may get

$$
\mathbf{0} \approx \sum_{\mathbf{x}_{B} \in \mathcal{S}_{\mathbf{x}_{A}}}-\nabla_{\mathbf{x}_{A}} W\left(\left|\mathbf{x}_{A}-\mathbf{x}_{B}\right|\right) V_{B}
$$

and

$$
\mathbf{I} \approx \sum_{\mathbf{x}_{B} \in \mathcal{S}_{\mathbf{x}_{A}}}-\mathbf{x}_{B} \otimes \nabla_{\mathbf{x}_{A}} W\left(\left|\mathbf{x}_{A}-\mathbf{x}_{B}\right|\right) V_{B}
$$

From Eq. (24) and (25), one can obtain

$$
\left\langle\nabla f\left(\mathbf{x}_{A}\right)\right\rangle=\sum_{\mathbf{x}_{B} \in \mathcal{S}_{\mathbf{x}_{A}}}\left[-f\left(\mathbf{x}_{A}\right) \nabla_{\mathbf{x}_{A}} W\left(\left|\mathbf{x}_{A}-\mathbf{x}_{B}\right|\right)+f\left(\mathbf{x}_{B}\right) \nabla_{\mathbf{x}_{B}} W\left(\left|\mathbf{x}_{A}-\mathbf{x}_{B}\right|\right)\right] V_{B}
$$

Notice $\nabla_{\mathbf{x}_{B}} W\left(\left|\mathbf{x}_{A}-\mathbf{x}_{B}\right|\right)=-\nabla_{\mathbf{x}_{A}} W\left(\left|\mathbf{x}_{A}-\mathbf{x}_{B}\right|\right)$ is the used during the derivation.

Combine Eq. (25) and (26), one may construct a corrected gradient operator

$$
\bar{\nabla}_{\mathbf{x}_{A}} W\left(\left|\mathbf{x}_{A}-\mathbf{x}_{B}\right|\right)=-\mathbf{M}_{\mathbf{x}_{A}}^{-1} \nabla_{\mathbf{x}_{A}} W\left(\left|\mathbf{x}_{A}-\mathbf{x}_{B}\right|\right)
$$

where the matrix $\mathbf{M}_{\mathbf{x}_{A}}$ is defined as

$$
\mathbf{M}_{\mathbf{x}_{A}}:=\sum_{\mathbf{x}_{B} \in \mathcal{S}_{\mathbf{x}_{A}}}\left(\mathbf{x}_{B}-\mathbf{x}_{A}\right) \otimes \nabla_{\mathbf{x}_{A}} W\left(\left|\mathbf{x}_{A}-\mathbf{x}_{B}\right|\right) V_{B}
$$

By substituting the corrected gradient operator into Eq. (27), one can get

$$
\left\langle\bar{\nabla} f\left(\mathbf{x}_{A}\right)\right\rangle=\sum_{\mathbf{x}_{B} \in \mathcal{S}_{\mathbf{x}_{A}}}\left[f\left(\mathbf{x}_{A}\right) \mathbf{M}_{\mathbf{x}_{A}}^{-1} \nabla_{\mathbf{x}_{A}} W\left(\left|\mathbf{x}_{A}-\mathbf{x}_{B}\right|\right)-f\left(\mathbf{x}_{B}\right) \mathbf{M}_{\mathbf{x}_{B}}^{-1} \nabla_{\mathbf{x}_{B}} W\left(\left|\mathbf{x}_{A}-\mathbf{x}_{B}\right|\right)\right] V_{B}
$$

With these basics, we directly write the discretized SPH equations of motion for the 
simulation of explosives as,

$$
\begin{aligned}
\frac{D \rho_{A}}{D t} & =\sum_{\mathbf{x}_{B} \in \mathcal{H}_{\mathbf{x}_{A}}} m_{B}\left(\mathbf{v}_{B}-\mathbf{v}_{A}\right) \cdot \bar{\nabla}_{\mathbf{x}_{B}} W\left(\left|\mathbf{x}_{B}-\mathbf{x}_{A}\right|\right) \\
\frac{D \mathbf{v}_{A}}{D t} & =-\sum_{\mathbf{x}_{B} \in \mathcal{H}_{\mathbf{x}_{A}}} m_{B}\left(\frac{p_{A}}{\rho_{A}^{2}}+\frac{p_{B}}{\rho_{B}^{2}}+\Pi_{A B}\right) \bar{\nabla}_{\mathbf{x}_{B}} W\left(\left|\mathbf{x}_{B}-\mathbf{x}_{A}\right|\right) \\
\frac{D e_{A}}{D t} & =\frac{1}{2} \sum_{\mathbf{x}_{B} \in \mathcal{H}_{\mathbf{x}_{A}}} m_{B}\left(\frac{p_{A}}{\rho_{A}^{2}}+\frac{p_{B}}{\rho_{B}^{2}}+\Pi_{A B}\right)\left(\mathbf{v}_{A}-\mathbf{v}_{B}\right) \cdot \bar{\nabla}_{\mathbf{x}_{B}} W\left(\left|\mathbf{x}_{B}-\mathbf{x}_{A}\right|\right) \\
\frac{D \mathbf{x}_{A}}{D t} & =\mathbf{v}_{A}
\end{aligned}
$$

where $\rho, \mathbf{v}, e, p$ are the density, velocity, internal energy and pressure at the corresponding particle. $\frac{D()}{d t}$ represents the time derivative of the quantity in the bracket. $\Pi_{A B}$ is the standard Monaghan viscosity [23], as shown in Eq. [11). $p=(\gamma-1) \rho e$ is the pressure resulting from a (TNT) explosive charge model. For details of these equations, the readers may consult [9].

\section{COUPLING SCHEME OF THE TWO METHODS}

Before proceeding to the details of the coupling, we want to mention that the state-based peridynamics is based on Lagrangian formulation and the SPH methodology is based on Eulerian formulation. Thus the influence function of the state based peridynamics $\omega\left(\left|\mathbf{X}_{A \rightarrow B}\right|\right)$ is a Lagrangian kernel and the smoothing function of the SPH method $W\left(\left|\mathbf{x}_{B}-\mathbf{x}_{A}\right|\right)$ is an Eulerian kernel. In the peridynamics-SPH computational domain, there is an interphase zone, consisting of both peridynamic and SPH particles. The coupling of peridynamics and SPH method mainly requires the related force and deformation transmissions over the interphase zone. In general, a Lagrangian kernel only exists for a peridynamic particle and an Eulerian kernel merely exists for an SPH particle. But for the purpose of the coupling, it is assumed that for any particle in the interphase zone, there are both a Lagrangian kernel and an Eulerian one. In this section, the following two 
passes of transmissions in the interphase zone are first discussed: (1) the contribution of an SPH particle to the governing equations of a peridynamic particle (see Eqs. (4) $-(10)$ ) and (2) the contribution of a peridynamic particle to the governing equations of an SPH particle (see Eqs. (31)-(34)). Then the choice of the smoothing function (for SPH) and influence function (for PD theory) are presented, by assuming the equivalence of the two methods in the current configuration.

\section{An SPH particle to a peridynamic particle}

For an SPH particle $\mathbf{X}_{B} \in \mathcal{H}_{\mathbf{X}_{A}}$, one can directly treat $\mathbf{X}_{B}$ as a peridynamic particle by constructing the same influence function $\omega\left(\left|\mathbf{X}_{A \rightarrow B}\right|\right)$, and the shape tensors $\mathbf{K}_{X_{B}}, \mathbf{N}_{X_{B}}$. Combine Eq. (4)-(10) and the force applying on a peridynamic particle can be written as

$$
\mathbf{f}_{A}=\sum_{\mathbf{X}_{B} \in \mathcal{H}_{\mathbf{x}_{A}}}\left[\omega\left(\left|\mathbf{X}_{A \rightarrow B}\right|\right) \mathbf{P}_{\mathbf{X}_{A}} \mathbf{K}_{\mathbf{X}_{A}}^{-1} \cdot \mathbf{X}_{A \rightarrow B}-\omega\left(\left|\mathbf{X}_{B \rightarrow A}\right|\right) \mathbf{P}_{\mathbf{X}_{B}} \mathbf{K}_{\mathbf{X}_{B}}^{-1} \cdot \mathbf{X}_{B \rightarrow A}\right] V_{B}^{0}
$$

For the SPH particle, the only quantity unknown is the first Piola-Kirchhoff stress tensor $\mathbf{P}_{\mathbf{X}_{A}}$, which does not exist in the hydrodynamics system that is based on Eulerian formulation. Luckily, at any SPH particle $\mathbf{X}_{B}$, there is a pressure $p_{B}$ that can be viewed as a Cauchy stress

$$
\sigma_{\mathbf{X}_{B}}=p_{B} \mathbf{I}
$$

where I is a second-order identity tensor. The Cauchy stress tensor can be transformed into the first Piola-Kirchhoff stress tensor by using the formula

$$
\mathbf{P}_{\mathbf{X}_{B}}=J_{B} \sigma_{\mathbf{X}_{B}} \mathbf{F}_{\mathbf{X}_{B}}^{-T}
$$

where $\mathbf{F}_{\mathbf{X}_{B}}$ is the deformation gradient at particle $\mathbf{X}_{B}$ and $J_{B}=\operatorname{det}\left[\mathbf{F}_{\mathbf{X}_{B}}\right]$.

Notice that the deformation gradient $\mathbf{F}_{\mathbf{X}_{B}}$ can be approximated by Eq. (10), using the corresponding Lagrangian kernel of the SPH particle $\mathbf{X}_{B}$. Practically, it might be easier to 
first construct the following two similar shape tensors using the Eulerian kernel. But the problem is that one can never guarantee that the matrix $\mathbf{K}_{\mathbf{X}_{B}}$ is always invertible (if the Eulerian kernel is adopted), given that one cannot control the number of active bonds in the current configuration.

\section{A peridynamic particle to an SPH particle}

For a peridynamic particle $\mathbf{x}_{B} \in \mathcal{S}_{\mathbf{x}_{A}}$, one needs to construct its corresponding SPH quantities, such as the density $\rho_{B}$, velocity $\mathbf{v}_{B}$ and pressure $p_{B}$, in order to account for its effect in the SPH governing Eqs. (31)-(34)). In fact, the velocity $\mathbf{v}_{B}$ is always available for a peridynamic particle. In addition, the density $\rho_{B}$ can be calculated as

$$
\rho_{B}=\frac{1}{J_{B}} \rho_{B}^{0}
$$

and the pressure $p_{B}$ can be obtained by

$$
p_{B}=\operatorname{tr}\left(\sigma_{B}\right)=\frac{1}{J_{B}} \operatorname{tr}\left(\mathbf{P}_{\mathbf{X}_{B}} \mathbf{F}_{\mathbf{X}_{B}}^{T}\right)
$$

where $\rho_{B}^{0}$ is the initial density at particle $\mathbf{X}_{B}$ and $J_{B}=\operatorname{det}\left[\mathbf{F}_{\mathbf{X}_{B}}\right]$.

\section{The kernel functions}

In the peridynamics-SPH coupling framework, there are two different types of kernel functions: the influence function $\omega\left(\left|\mathbf{X}_{B}-\mathbf{X}_{A}\right|\right)$ (for PD theory) and the smoothing function $W\left(\left|\mathbf{x}_{B}-\mathbf{x}_{A}\right|\right)$ (for SPH methodology), which are defined in the horizon $\mathcal{H}_{\mathbf{x}_{A}}$ and supporting domain $\mathcal{S}_{\mathbf{x}_{A}}$, respectively.

In the original work of peridynamics, the only requirement for the influence function $\omega\left(\left|\mathbf{X}_{B}-\mathbf{X}_{A}\right|\right)$ is to be zero outside the family $\mathcal{H}_{\mathbf{X}^{A}}$ and nonzero inside, that is

$$
\left\{\begin{array}{l}
\omega\left(\left|\mathbf{X}_{B}-\mathbf{X}_{A}\right|\right)=0,\left|\mathbf{X}_{B}-\mathbf{X}_{A}\right|>\delta_{\mathbf{X}^{A}} \\
\omega\left(\left|\mathbf{X}_{B}-\mathbf{X}_{A}\right|\right) \neq 0, \text { otherwise }
\end{array}\right.
$$


In contrast, a smoothing function has to satisfy a number of conditions, such as the compact condition, the unity condition, and the delta function property [21]. Mathematically, these three conditions can be expressed as

$$
\left\{\begin{array}{l}
W\left(\left|\mathbf{x}_{B}-\mathbf{x}_{A}\right|\right)=0, \text { for }\left|\mathbf{x}_{B}-\mathbf{x}_{A}\right|>h \\
\lim _{h \rightarrow 0} W\left(\left|\mathbf{x}_{B}-\mathbf{x}_{A}\right|\right)=\delta\left(\mathbf{x}_{B}-\mathbf{x}_{A}\right) \\
\int_{\mathcal{S}_{\mathbf{x}_{A}}} W\left(\left|\mathbf{x}_{B}-\mathbf{x}_{A}\right|\right) d V_{\mathbf{x}_{B}}=1
\end{array}\right.
$$

In the interphase zone, an SPH particle within the horizion of a peridynamic particle needs an influence function, so as to provide the right contribution. Therefore, it might be of great significance that the right influence function and smoothing kernel function are selected in the whole framework. In this work, the shape and size of the horizon and that of the supporting domain are chosen to be the same.

To better characterize the form of the two different kernels, we would like to look at the force density of PD and the SPH formulation, both in the current configuration. In classical continuum mechanics, without considering body forces, the equations of motion is

$$
\rho \ddot{\mathbf{u}}=\nabla_{\mathbf{x}} \cdot \sigma
$$

Following Eq. (30), in the SPH formulation, the force vector applying on particle $\mathbf{x}_{A}$ can be written as

$$
\begin{aligned}
\mathbf{f}_{A}^{S P H} & =\sum_{\mathbf{x}_{B} \in \mathcal{S}_{\mathbf{x}_{A}}}\left[\sigma_{\mathbf{x}_{A}} \mathbf{M}_{\mathbf{x}^{A}}^{-1} \cdot \nabla_{\mathbf{x}_{A}} W\left(\left|\mathbf{x}_{A}-\mathbf{x}_{B}\right|\right)-\sigma_{\mathbf{x}_{B}} \mathbf{M}_{\mathbf{x}_{B}}^{-1} \cdot \nabla_{\mathbf{x}_{B}} W\left(\left|\mathbf{x}_{A}-\mathbf{x}_{B}\right|\right)\right] V_{B} \\
& =\sum_{\mathbf{x}_{B} \in \mathcal{S}_{\mathbf{x}_{A}}}-\frac{1}{x_{A \rightarrow B}} \frac{\partial W\left(\left|\mathbf{x}_{A}-\mathbf{x}_{B}\right|\right)}{\partial x_{A \rightarrow B}}\left(\sigma_{\mathbf{x}_{A}} \mathbf{M}_{\mathbf{x}_{A}}^{-1} \cdot \mathbf{x}_{A \rightarrow B}-\sigma_{\mathbf{x}_{B}} \mathbf{M}_{\mathbf{x}_{B}}^{-1} \cdot \mathbf{x}_{B \rightarrow A}\right) V_{B}
\end{aligned}
$$

where

$$
\mathbf{M}_{\mathbf{x}_{A}}=\sum_{\mathbf{x}^{B} \in \mathcal{S}_{\mathbf{x}^{A}}}\left(-\frac{1}{x_{A \rightarrow B}} \frac{\partial W\left(\left|\mathbf{x}_{A}-\mathbf{x}_{B}\right|\right)}{\partial x_{A \rightarrow B}} \mathbf{x}_{A \rightarrow B} \otimes \mathbf{x}_{A \rightarrow B}\right) V_{B}
$$


Notice that we can also derive the force vector in Eq. (35) for the PD theory based on the current configuration,

$$
\mathbf{f}_{A}^{P D}=\sum_{\mathbf{x}_{B} \in \mathcal{H}_{\mathbf{x}_{A}}}\left[\omega\left(\left|\mathbf{x}_{A \rightarrow B}\right|\right) \sigma_{\mathbf{x}_{A}} \mathbf{K}_{\mathbf{x}_{A}}^{-1} \cdot \mathbf{x}_{A \rightarrow B}-\omega\left(\left|\mathbf{x}_{B \rightarrow A}\right|\right) \sigma_{\mathbf{x}_{B}} \mathbf{K}_{\mathbf{x}_{B}}^{-1} \cdot \mathbf{x}_{B \rightarrow A}\right] V_{B}
$$

Compare the force vector applying on a peridynamic particle (see Eq. (45)) with the that acting on an SPH particle (see Eq. (43)), one can see that if we choose

$$
\begin{aligned}
& \mathcal{H}_{\mathbf{x}^{A}}=\mathcal{S}_{\mathbf{x}^{A}} \\
& \omega\left(\left|\mathbf{x}_{A \rightarrow B}\right|\right)=-\frac{1}{x_{A \rightarrow B}} \frac{\partial W\left(\left|\mathbf{x}_{A}-\mathbf{x}_{B}\right|\right)}{\partial x_{A \rightarrow B}}
\end{aligned}
$$

then one would have

$$
\mathbf{K}_{\mathbf{x}_{A}}=\mathbf{M}_{\mathbf{x}_{A}}
$$

and

$$
\mathbf{f}_{A}^{P D}=\mathbf{f}_{A}^{S P H}
$$

Therefore, in the PD-SPH coupling domain, it might be better to choose the horizon, influence function in PD theory and the supporting domain, smoothing kernel function in SPH method such that the conditions in Eqs. (46) and (47) are satisfied. For instance, if the SPH kernel function is

$$
W(x)=\frac{1}{\left(\pi h^{2}\right)^{n / 2}} \exp \left(-x^{2} / h^{2}\right),
$$

then the PD influence function should be of the form

$$
\omega(x)=-\frac{1}{x} \frac{d W(x)}{d x}=\frac{2}{h^{2}\left(\pi h^{2}\right)^{n / 2}} \exp \left(-x^{2} / h^{2}\right) .
$$

where $n$ is dimension of the problem and $h$ is the smoothing length (or horizon size). 


\section{SoIL MODELING}

\section{Drucker-Prager plasticity model}

The state-based peridynamics can be termed as a continuum theory in an integral form, owing to the fact that it can incorporate classical stress-strain models through the force state (see Eq. (5)) that is based on an equivalence of the strain energy density function. Soils usually present great nonlinearities after plastic yielding, with pressure-sensitive yield surfaces. In the literature, there are several successful constitutive models for soils, such as the Drucker-Prager (DP) model, the Mohr-Coulomb (MC) model and the Matsuoka-Nakai (MN) model. In this work, the Drucker-Prager (DP) plasticity model [13] is adopted to characterize the plastic behaviors of the soil. The DP model has been extensively used in numerical simulations of soil, rock and concrete structures [15]. The material parameters of the DP model for soils are well documented with experimental calibrations. In this section, a brief review of the DP model is first offered, mainly focusing on the constitutive updates for a nonlinear finite deformation, which are then expressed in the corresponding peridynamics formulations.

The yield function of the DP model is given as

$$
\left\{\begin{array}{l}
f=\|\mathbf{s}\|-\left(A\left(\phi^{\prime}\right) c^{\prime}-B\left(\phi^{\prime}\right) p^{\prime}\right) \leq 0 \\
A\left(\phi^{\prime}\right)=\frac{2 \sqrt{6} \cos \phi^{\prime}}{3+\beta \sin \phi^{\prime}} \\
B\left(\phi^{\prime}\right)=\frac{2 \sqrt{6} \sin \phi^{\prime}}{3+\beta \sin \phi^{\prime}},-1 \leq \beta \leq 1
\end{array}\right.
$$

where $\mathbf{s}$ is the deviatoric stress tensor, $\mathrm{c}^{\prime}$ the effective cohesion $(\mathrm{Pa}), \mathrm{p}^{\prime}$ the mean effective stress and $\phi^{\prime}$ the effective friction angle. $\beta=1$ and $\beta=-1$ approximates two cases of the DP model, i.e, the triaxial extension (TE) corners and the triaxial compression (TC) corners of the MC yield surface, respectively [24]. For a non-associative flow, the DP 
plastic potential function can be expressed as

$$
\left\{\begin{array}{l}
g=\|\mathbf{s}\|-\left(A\left(\psi^{\prime}\right) c^{\prime}-B\left(\psi^{\prime}\right) p^{\prime}\right) \\
A\left(\psi^{\prime}\right)=\frac{2 \sqrt{6} \cos \psi^{\prime}}{3+\beta \sin \psi^{\prime}} \\
B\left(\psi^{\prime}\right)=\frac{2 \sqrt{6} \sin \psi^{\prime}}{3+\beta \sin \psi^{\prime}},-1 \leq \beta \leq 1
\end{array}\right.
$$

where $\psi^{\prime}$ is the effective dilation angle. If $\phi^{\prime}=\psi^{\prime}=0$, Eqs. (52) and (53) are the identical (the yield function and potential function overlaps), and the DP model reduces to a form similar to $J_{2}$ plasticity, which is very useful in approximating the undrained shear strength of soil. The Helmholtz free energy function $\rho_{s} \Psi$ per unit deformed soil volume can be decomposed into elastic and plastic parts as,

$$
\rho_{s} \Psi\left(\epsilon^{e}, \zeta\right)=\frac{1}{2} \epsilon^{e}: \mathbf{D}^{e}: \epsilon^{e}+\frac{1}{2} \zeta \cdot \mathbf{H} \cdot \zeta
$$

where $\epsilon^{e}$ is the elastic strain tensor, $\mathbf{D}^{e}$ the elastic modulus tensor, $\mathbf{H}$ the hardening/softening modulus matrix and $\zeta$ a set of strain-like internal state variables (ISVs) associated with plastic hardening/softening, representing the evolution of the underlying soil microstructure phenomenologically related to the experimentally-observed behavior. With the Helmholtz free energy in Eq. (54), the rate equations of stress $\left(\sigma^{\prime}\right)$ and stress-like ISVs $\left(\mathbf{q}^{\zeta}\right)$ can be derived as,

$$
\begin{aligned}
\dot{\boldsymbol{\sigma}}^{\prime} & =\frac{\partial\left(\rho_{s} \Psi\right)}{\partial \dot{\boldsymbol{\epsilon}}^{e}}=\mathbf{D}^{e}: \dot{\boldsymbol{\epsilon}}^{e}=\mathbf{D}^{e}:\left(\dot{\boldsymbol{\epsilon}}-\dot{\boldsymbol{\epsilon}}^{p}\right) \\
\dot{\mathbf{q}}^{\zeta} & =\frac{\partial\left(\rho_{s} \Psi\right)}{\partial \dot{\zeta}}=\mathbf{H} \cdot \dot{\zeta}
\end{aligned}
$$

where $\boldsymbol{\epsilon}^{p}$ is the plastic strain tensor and $\mathbf{q}^{\zeta}$ are the specific internal states $\mathbf{q}^{\zeta}=\left\{c^{\prime}, \phi^{\prime}, \psi^{\prime}\right\}^{T}$. To the sake of simplicity, it is assumed that the ISVs are to evolve under an independent, linear hardening/softening model, i.e.,

$$
\mathbf{H}=\left[\begin{array}{ccc}
H^{c} & 0 & 0 \\
0 & H^{\phi} & 0 \\
0 & 0 & H^{\psi}
\end{array}\right]
$$


where $H^{c}, H^{\phi}, H^{\psi}$ are the linear hardening/softening moduli for $c^{\prime}, \phi^{\prime}, \psi^{\prime}$, respectively. Based on the plastic potential function (see Eq. (53)), the evolution of plastic flow can be derived as

$$
\begin{aligned}
\dot{\boldsymbol{\epsilon}}^{p} & =\dot{\gamma} \frac{\partial g}{\partial \sigma^{\prime}}=\dot{\gamma}\left(\frac{\partial\|\mathbf{s}\|}{\partial \sigma^{\prime}}+B\left(\psi^{\prime}\right) \frac{\partial p^{\prime}}{\partial \sigma^{\prime}}\right) \\
& =\dot{\gamma}\left(\frac{\mathbf{s}}{\|\mathbf{s}\|}+\frac{1}{3} B\left(\psi^{\prime}\right) \mathbf{I}\right)
\end{aligned}
$$

where $\mathbb{I}$ is the second order identity tensor. The evolution equations of the ISVs are defined as,

$$
\begin{aligned}
\dot{\mathbf{q}}^{\zeta} & =\dot{\gamma} \mathbf{H} \cdot \mathbf{h}\left(\sigma, \mathbf{q}^{\zeta}\right) \\
\mathbf{h} & =-\frac{\partial f}{\partial \mathbf{q}^{\zeta}},
\end{aligned}
$$

where $\mathbf{h}$ is a hardening function. Using the pricinple of maximum plastic dissipation, one can get

$$
\mathbf{h}=\left(\begin{array}{l}
A\left(\phi^{\prime}\right) \\
\frac{\partial A\left(\phi^{\prime}\right)}{\partial \phi^{\prime}} c^{\prime}-\frac{\partial B\left(\phi^{\prime}\right)}{\partial \phi^{\prime}} p^{\prime} \\
\frac{\partial A\left(\psi^{\prime}\right)}{\partial \psi^{\prime}} c^{\prime}-\frac{\partial B\left(\psi^{\prime}\right)}{\partial \psi^{\prime}} p^{\prime}
\end{array}\right)
$$

Using the consistency condition $\dot{f}=0$, the plastic multiplier $\dot{\gamma}$ can be obtained as

$$
\left\{\begin{array}{l}
\dot{\gamma}=\frac{1}{\chi} \frac{\partial f}{\partial \sigma^{\prime}}: \mathbf{D}^{e}: \dot{\boldsymbol{\epsilon}} \\
\chi=\frac{\partial f}{\partial \sigma^{\prime}}: \mathbf{D}^{e}: \frac{\partial g}{\partial \sigma^{\prime}}-\frac{\partial f}{\partial \mathbf{q}^{\zeta}}: \mathbf{H} \cdot \mathbf{h}
\end{array}\right.
$$

Eqs. (55), (56) and (61) are the equations of constitutive update of a non-linear plastic constitutive model. The three sets of unknowns $\left(\sigma, \mathbf{q}^{\zeta}, \dot{\gamma}\right)$ are coupled in the non-linear evolution equations, which can be solved by the Newton-Raphson iteration method [25]. For simplicity, the following explicit update algorithm is adopted in this work,

$$
\begin{aligned}
\sigma_{n+1}^{\prime, t r} & =\sigma_{n}^{\prime}+\mathbf{D}^{e}: \Delta \boldsymbol{\epsilon} \\
f_{n+1}^{t r} & =\left\|\mathbf{s}^{t r}\right\|-\left[A\left(\phi_{n}^{\prime}\right) c_{n}^{\prime}-B\left(\phi_{n}^{\prime}\right)\left(p^{\prime}\right)_{n+1}^{t r}\right]
\end{aligned}
$$


where the supscript $t r$ stands for the corresponding trial items.

if $f_{n+1}^{t r}<0$, elastic phase: update $\sigma_{n+1}^{\prime}=\sigma_{n+1}^{\prime, t r}$ and $\mathbf{q}_{n+1}^{\zeta}=\mathbf{q}_{n}^{\zeta}$.

if $f_{n+1}^{t r} \geq 0$, plastic phase:

$$
\begin{aligned}
\Delta \gamma & =\frac{f_{n+1}^{t r}}{2 \mu+K B\left(\phi^{\prime}\right) B\left(\psi^{\prime}\right)+H^{c}\left(A\left(\phi^{\prime}\right)\right)^{2}} \\
\sigma_{n+1}^{\prime} & =\sigma_{n+1}^{\prime, t r}-\Delta \gamma\left(K B\left(\psi^{\prime}\right) \mathbf{I}+2 \mu \hat{\mathbf{n}}_{n+1}\right) \\
\mathbf{q}_{n+1}^{\zeta} & =\mathbf{q}_{n}^{\zeta}+\Delta \gamma \mathbf{H} \cdot \mathbf{h}\left(\sigma, \mathbf{q}^{\zeta}\right)
\end{aligned}
$$

The trial stress is calculated based on Eq. (62), under the assumption of small deformation. But in general, the soil under blast loading shall undergo finite deformation with large rotations. Thus it should be replaced with a nonlinear one accounting for finite deformation. In this work, the nonlinear equations of the Hughes-Winget algorithm [26] are adopted.

In the Hughes-Winget algorithm, an intermediate configuration at time step $n+\alpha$ is defined

$$
\mathbf{x}^{n+\alpha}=(1-\alpha) \mathbf{x}^{n}+\alpha \Delta \mathbf{u}
$$

where $\alpha$ is a scalar and it is set to be 0.5 , without the loss of generality. Following the derivation of the approximated deformation gradient in Eq. (10), the deformation gradient at the configuration $\mathbf{x}_{n+\alpha}$ can be represented as,

$$
\mathbf{F}^{n+\alpha}=\frac{\partial \mathbf{x}^{n+\alpha}}{\partial \mathbf{X}}=\left(\sum_{\mathbf{x}_{B} \in \mathcal{H}_{\mathbf{x}_{A}}} \omega(|\boldsymbol{\xi}|)\left(\mathbf{x}_{B}^{n+\alpha}-\mathbf{x}_{A}^{n+\alpha}\right) \otimes \mathbf{X}_{A \rightarrow B}\right) \cdot \mathbf{K}_{\mathbf{x}_{A}^{-1}}^{-1}
$$

In addition, the gradient of $\Delta \mathbf{u}$ with respect to the reference configuration can be written as,

$$
\nabla_{\mathbf{x}} \mathbf{\Delta} \mathbf{u}=\frac{\partial(\Delta \mathbf{u})}{\partial \mathbf{X}}=\left(\sum_{\mathbf{X}_{B} \in \mathcal{H}_{\mathbf{x}_{A}}} \omega(|\boldsymbol{\xi}|)\left(\Delta \mathbf{u}_{B}-\Delta \mathbf{u}_{A}\right) \otimes \mathbf{X}_{A \rightarrow B}\right) \cdot \mathbf{K}_{\mathbf{X}_{A}}^{-1}
$$


Using the chain rule, one can then get deformation gradient increment

$$
\mathbf{G}=\frac{\partial(\Delta \mathbf{u})}{\partial \mathbf{x}_{n+\alpha}}=\nabla_{\mathbf{x}} \Delta \mathbf{u} \cdot \mathbf{F}_{n+\alpha}^{-1}
$$

which can be split into the following strain and rotation increments,

$$
\begin{aligned}
& \boldsymbol{\gamma}=\left(\mathbf{G}+\mathbf{G}^{T}\right) / 2 \\
& \boldsymbol{\omega}=\left(\mathbf{G}-\mathbf{G}^{T}\right) / 2
\end{aligned}
$$

The objective effective stress increment can then be evaluated as,

$$
\Delta \sigma^{\prime}=\mathbf{D}^{e}: \gamma
$$

In the end, Eq. (62) is replaced by the following set of equations

$$
\begin{aligned}
\sigma_{n+1}^{\prime} & =\hat{\sigma}_{n}^{\prime}+\Delta \boldsymbol{\sigma}^{\prime} \\
\hat{\sigma}_{n}^{\prime} & =\mathbf{\Omega}^{T} \cdot \boldsymbol{\sigma}_{n}^{\prime} \cdot \mathbf{\Omega} \\
\mathbf{\Omega} & =\mathbf{I}+(\mathbf{I}-\alpha \boldsymbol{\omega})^{-1} \cdot \boldsymbol{\omega} .
\end{aligned}
$$

\section{Adaptive dynamic relaxation}

In practical application, it is very important that a numerical solution can be verified by experiment or reliable computational result. But in general, experiments are conducted under static condition. Thus the Adaptive Dynamic Relaxation (ADR) is adopted [27], in which an artificial damping is introduced into the system, such that the steady-state (static) solution is retained. To determine the most effective damping effect, the damping coefficient is changed adaptively in each time step. In specific, by introducing the inertia and damping terms, the peridynamics governing equations (4) become

$$
\Lambda \ddot{\mathbf{U}}(\mathbf{X}, t)+c \mathbf{\Lambda} \dot{\mathbf{U}}(\mathbf{X}, t)=\int_{\mathcal{H}} \mathbf{f}\left(\mathbf{U}, \mathbf{U}^{\prime}, \mathbf{X}, \mathbf{X}^{\prime}\right) d V^{\prime},
$$


where $\Lambda$ is the fictitious diagonal density matrix $\lambda_{A A}=\rho_{A}$. $\mathbf{U}$ is the displacement vector of the particles and $\mathbf{f}\left(\mathbf{U}, \mathbf{U}^{\prime}, \mathbf{X}, \mathbf{X}^{\prime}\right)$ is the summation of the internal and external forces. The $A^{\text {th }}$ components of $\mathbf{U}$ and its force state are,

$$
\begin{aligned}
\mathbf{U}_{A} & =\mathbf{u}\left(\mathbf{x}_{A}, t\right) \\
\mathbf{F}_{A} & =\mathbf{b}\left(\mathbf{x}_{A}, t\right)+\frac{1}{\rho_{A}^{0}} \int_{\mathcal{H}_{\mathbf{x}_{A}}}\left[\underline{\mathbf{T}}\left(\mathbf{X}_{A}, \mathbf{X}_{A \rightarrow B}\right)-\underline{\mathbf{T}}\left(\mathbf{X}_{B}, \mathbf{X}_{B \rightarrow A}\right)\right] d V_{\mathbf{X}_{B}}
\end{aligned}
$$

The damping coefficient $c$ are computed every time step to obtain the fastest path to the steady-state solution. It is determined by the Rayleigh's quotient

$$
c_{n}=2 \sqrt{\left(\left(\mathbf{U}^{n}\right)^{T} \mathbf{K}^{n} \mathbf{U}^{n}\right) /\left(\left(\mathbf{U}^{n}\right)^{T} \mathbf{U}^{n}\right)},
$$

in which, $\mathbf{K}^{n}$ is the diagonal localized stiffness matrix, given as

$$
K_{i i}^{n}=-\left(F_{i}^{n} / \lambda_{i i}-F_{i}^{n-1} / \lambda_{i i}\right) /\left(\Delta t \dot{u}_{i}^{n-1 / 2}\right)
$$

where the superscript $n$ denotes the $n$-th time step and there is no summation for the subscript $i$. Interested readers may consult [27] for more details.

\section{NumericAl EXAMPLES}

\section{Verification of the soil constitutive in the PD theory}

In this example, numerical tests have been carried out to validate the peridynamics implementation of the Drucker-Prager (DP) model for soils. As shown in Fig. 1. we are considering a soil column with a square cross-section of side length $L=1 \mathrm{~m}$ and height $H=10 \mathrm{~m}$. The soil column is discretized into 10,300 particles. The column is subjected to a pressure load $f(t)$ on the top boundary. Both the step and harmonic loading functions are considered, as shown in Fig. 2. The other five boundaries are fixed with zero normal displacements. The simulation time step $\Delta t$ is set to be $0.0001 \mathrm{~s}$ and the duration of the 


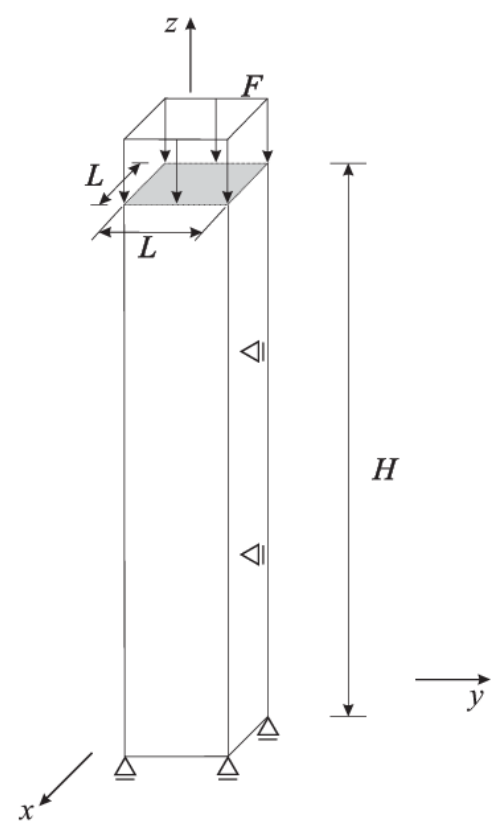

Figure 1: Configuration of the soil column.

\begin{tabular}{|c|c|c|c|c|c|}
\hline$E$ & $v$ & $\phi$ & $\psi$ & $c$ & $\beta$ \\
\hline $98,454,200 \mathrm{~Pa}$ & 0.25 & 0.738663 & 0.738663 & $208,848 \mathrm{~Pa}$ & -1 \\
\hline
\end{tabular}

Table 1: Material parameters of Drucker-Prager model.

simulation is $t=10 \mathrm{~s}$. The material parameters of the Drucker-Prager model are given in Table 1, which are taken from [16]. The Adaptive Dynamic Relaxation method is employed to obtain the static solutions, which can then be used to compare with the static finite element solutions from ANSYS software.

Fig. 3 shows the vertical displacements at the top of the column for three different load magnitudes all converge to the finite element solutions (ANSYS). As shown in Table 2 . The relative errors for all the three cases are less than $1.2 \%$. 


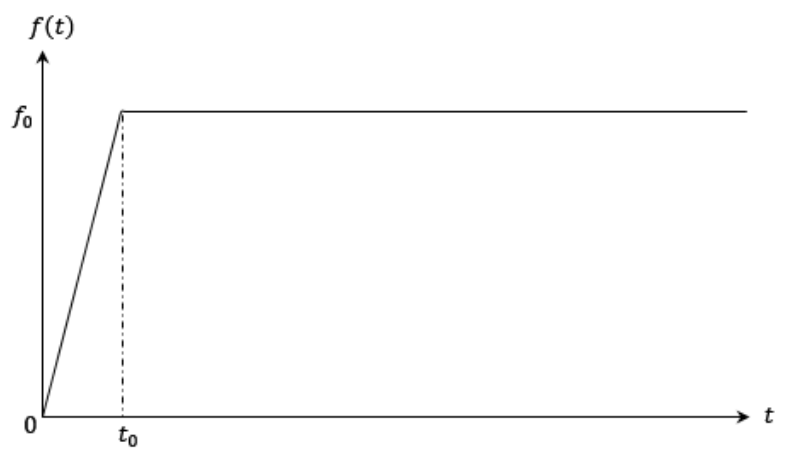

Figure 2: Step loading function

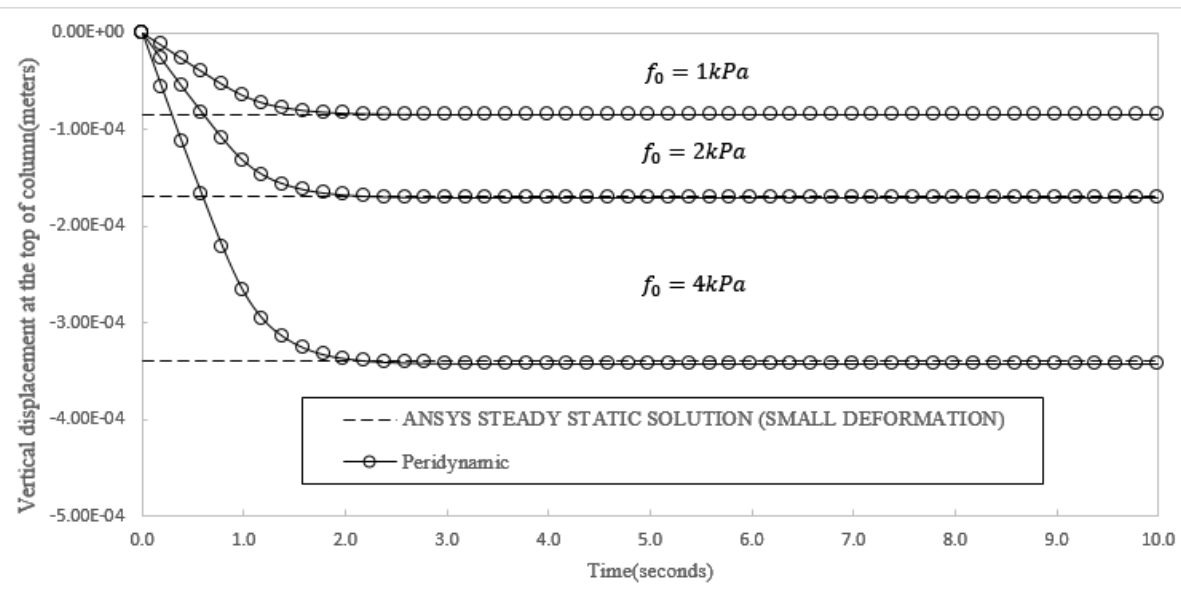

Figure 3: Convergence of the peridynamic results to the FEM solutions

\begin{tabular}{ccccc}
\hline Vertical Displacement & & \multicolumn{3}{c}{ Value } \\
\cline { 1 - 1 } \cline { 5 - 5 } Load(kPa) & & 1 & 2 & 4 \\
\cline { 1 - 4 } \cline { 5 - 6 } ANSYS solution(m) & & $0.846 \mathrm{E}-4$ & $0.169 \mathrm{E}-3$ & $0.339 \mathrm{E}-3$ \\
Peridynamic solution(m) & $0.856 \mathrm{E}-4$ & $0.171 \mathrm{E}-3$ & $0.342 \mathrm{E}-3$ \\
Relative Error(\%) & & 1.18 & 1.18 & 0.885 \\
\hline
\end{tabular}

Table 2: Comparison between DP peridynamics and ANSYS results 


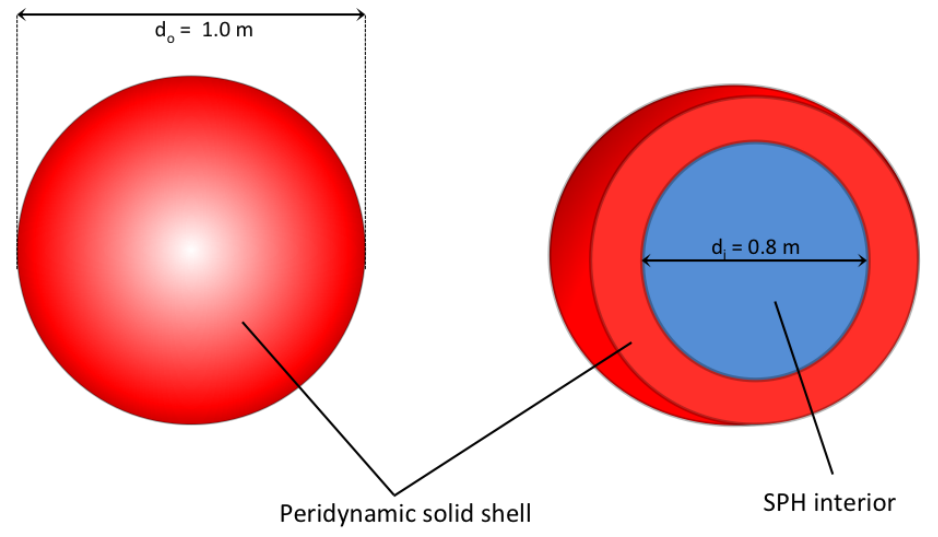

Figure 4: Layout of Spherical shell (left) and cut through mid-section (right).

\section{Validation of the peridynamics-SPH coupling scheme}

In this section, we present a simple benchmark problem of an expanding spherical shell composed of peridynamic solid particles and SPH gas particles in the interior.

In the proposed coupling alogrithm, particles in the peridynamics-SPH interphase zone will experience forces from both methods. In order to ensure consistency between the two methods, the pressure imposed by SPH particles is converted to an equivalent peridynamic force state, and vice versa. The main objective in this simulation is to verify that as the SPH gas expands, the forces will transfer correctly to the peridynamic particles and will produce stresses comparable to the corresponding analytical solution.

The geometry consists of a rubber sphere with an outer diameter of $1 m$ and inner diameter of $0.8 \mathrm{~m}$. Both the peridynamic and SPH particles are spaced at $\Delta=0.1 \mathrm{~m}$ apart in the $x, y$, and $z$ directions. The horizon size of the peridynamic particles is $\delta=3 \Delta=0.3 m$. The rubber's assigned material parameters include a young's modulus of 50MPa, a poisson's ratio of 0.499 (nearly incompressible), and a density of $1200 \frac{\mathrm{kg}}{\mathrm{m}^{3}}$. This layout is shown in Fig. 4 . 
For simplicity, we consider a quasi-static uniform pressure loading and infinitesimal displacements. To accommodate these considerations in the simulation, we allow the SPH particles' pressure, velocity, and acceleration to update until reaching an average (within the $\mathrm{SPH}$ domain) value of $25 \mathrm{kPa}$. Once the average pressure value is reached, the physical variables of the SPH particles are maintained such that the pressure is held constant at $25 \mathrm{kPa}$ for the remainder of the simulation.

As the internal pressure increases to its maximum value, the forces imposed by the SPH particles will transfer to the peridynamic solid shell particles. In this process, the peridynamic particles will oscillate about the steady-state solution. in order to show a clear convergence to a steady-state solution, a damping term was added to the external force vector of the peridynamic particles. Note that the addition of this extra term does not change the final value of the steady-state solution, it simply dissipates the motion of the particles thus providing a quasi-static solution.

The computed stress for both SPH and peridynamic particles considers the average of the diagonal terms of the Cauchy stress tensor $\sigma$ (the components represent stress in the standard Cartesian coordinates, i.e. $\sigma_{x x}, \sigma_{y y}$, and $\sigma_{z z}$ ). In both cases, the off-diagonal terms were ignored since they were comparatively negligible. As illustrated in figure 5 the average stresses of the peridynamic particles corresponds very closely to the imposed pressure by the SPH particles once the oscillations dissipate and the peridynamic stresses reach steady-state. This result indicates that the physics translates accurately between $\mathrm{SPH}$ and peridynamics. In other words, converting between peridynamic force states and SPH pressures (and vice versa) will provide a steady-state equilibrium solution in the quasi-static case.

With the given geometric parameters, the shell can be viewed analytically as a thick 


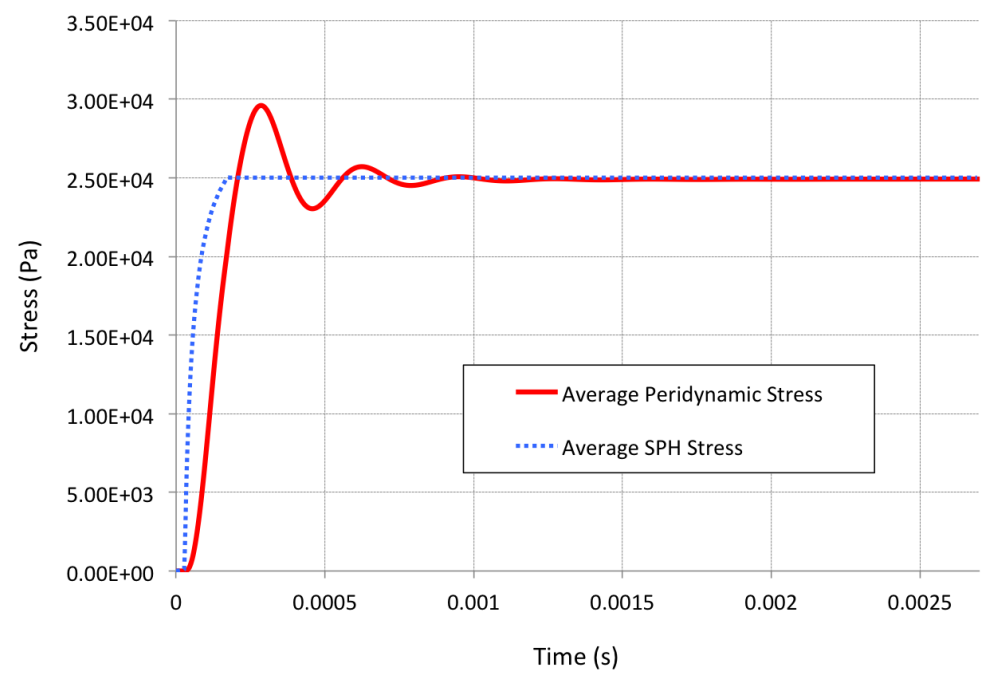

Figure 5: Average stress vs. time of an expanding peridynamic spherical shell due to internal pressure from SPH particles.

\section{sp}

spherical membrane whose hoop stresses may be computed using the following equation

$$
\sigma_{\theta \theta}=\frac{p d_{i}^{3}}{d_{o}^{3}-d_{i}^{3}}\left(\frac{d_{o}^{4}}{2 r^{3}}+1\right) d_{i} \leq r \leq d_{o}
$$

where $p$ is the average internal pressure, $d_{0}$ and $d_{i}$ are the inner and outer diameters of the sphere (respectively), and $r$ is the position of interest along the shell. In our configuration, the inner diameter is set as $0.8 \mathrm{~m}$. This means that any particle whose distance is less than $0.8 m$ from the center of the sphere is an SPH particle. However, since our grid consists of meshless particles, the actual boundary between the peridynamic and sph particles may lie anywhere in between the assigned value of $0.8 m$ and $0.8 m-2 \Delta=0.6 m$. In order to validate our numerical results with the analytical results obtained from Eq. (82), we will use the average stress value from the simulation to back-calculate a value of the shell's inner diameter. For two given shell particles at the coordinates $(0,0,0.40)$ (particle A) and $(0,0,0.50)$ (Particle B), the computed hoop stresses are 29,878 $\mathrm{kPa}$ and 15,311 $\mathrm{kPa}$ 


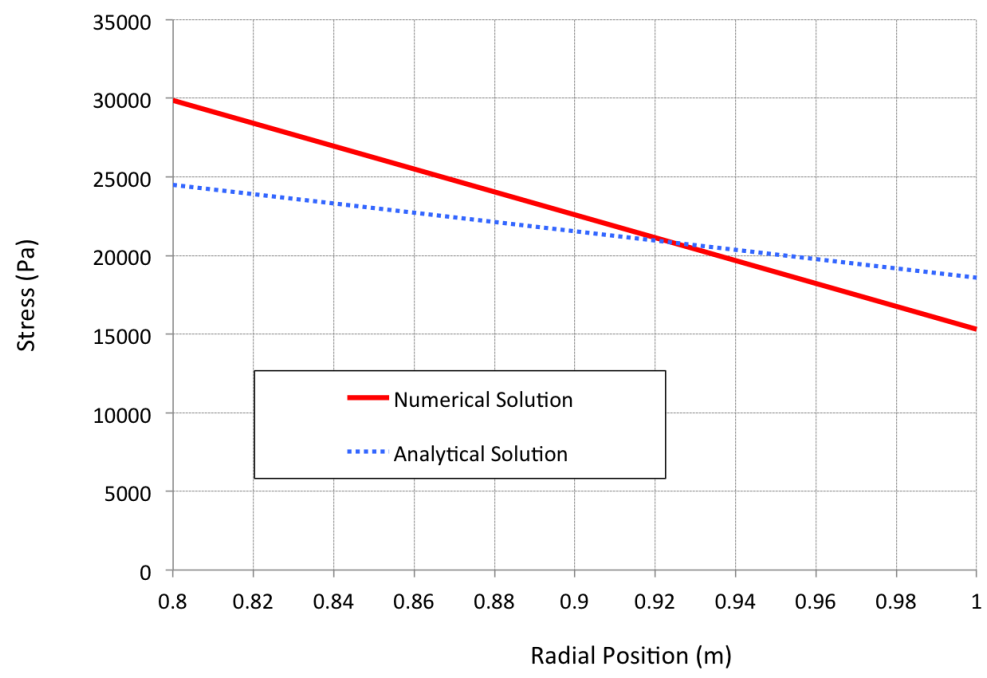

Figure 6: Relation between numerical and analytical values of the hoop stress along the radial position of the peridynamic spherical shell.

(respectively). Plugging the value of $r$ that corresponds to the particle coordinates, the computed hoop stress, and an outer diameter of $1 m$ into equation Eq. (82), we obtain a corresponding inner diameter of $0.72 \mathrm{~m}$ and $0.66 \mathrm{~m}$ for particles A and B respectively. Since both of these values lie within the range of possible inner diameters, we can conclude that they correspond to the analytical solution for a thick-walled sphere.

We can compute the analytical hoop stress along the radius of the shell assuming that the inner sphere diameter is $0.692 \mathrm{~m}$, which is the average of the calculated values of particle A and B. As shown in Fig. 6, the analytical solution produces comparable hoop stress values to those provided by the numerical simulation.

\section{Soil fragmentation under blast loading}

In this example, a numerical simulation is conducted to test the capability of peridynamics to capture shock wave induced soil fragmentations caused by blast loads of the buried 

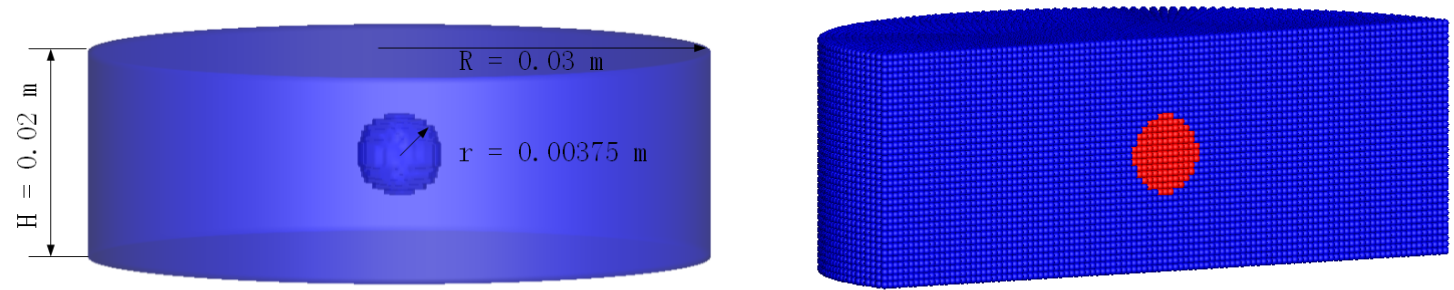

(a)

(b)

Figure 7: A soil cylinder with a spherical TNT bomb: (a) geometrical configuration; (b) representation of the domain with 327,709 discrete particles, of which 326,616 are peridynamic particles (blue) and 1093 are SPH particles (red)

explosive. In specific, we are considering a flat three-dimensional soil cylinder, with a spherical TNT bomb located right at the center. As shown in Fig. 7(a), the dimension of the soil cylinder is $R \times h=0.03 m \times 0.02 m$ and the radius of the spherical bomb at the center is $r=0.00375 \mathrm{~m}$. The motivation for this simulation is to predict soil fragmentation/ejection caused by shock waves generated from buried explosives. As shown in Fig. 7(b), the whole domain is discretized into 327,709 particles, consisting of 326,616 peridynamic particles (blue) and $1093 \mathrm{SPH}$ particles (red). In fact, the soil cylinder is first discretized by an FEM mesh, with 327,709 nodes and 314,928 hexahedron elements, which are then used to generate the particle positions (nodes), associated volumes and their corresponding horizon sizes $\delta \mathbf{X}$ or smoothing lengths $h_{\mathbf{x}}$. The SPH particles represent the computational domain of the spherical TNT bomb, and the peridynamic particles represent the rest of the cylinder domain. The few layers of particles at the bottom of the cylinder are fixed with zero displacements in the vertical direction through out the 
simulation, so that there is no particle penetrating through the bottom. Meanwhile, no gravitational force is considered in the simulation. The whole system is subjected to the perdynamic-SPH particle interaction, together with the artificial viscosity (to avoid particle inter-penetraction) and the essential boundary at the bottom. The material parameters of the DP model are the same as those in Tables 1 . The simulation time step is $\Delta t=6.125 \times 10^{-8}$ s. The initial energy of the SPH particles (denotation energy per unit mass) is $e_{0}=4.29 \times 10^{6} \mathrm{~J} / \mathrm{Kg}$, which is well documented in the literature [9]. Time sequences of the dynamic soil fragmentation/ejection processes are presented in Fig. 8 (view from the top) and 9 (view from a vertical cross section).

From Fig. 8, one can see that due to the blast of the buried TNT (SPH particles), peridynamic particles close to the top of the cylinder are pushed up and form a spherical dome. In the beginning, the dome is very shallow (see Fig. $8(a-c))$ and it then becomes larger and larger (see Fig. 8 (d-f)). This is easy to understand, because the outmost peridynamic particles in the top of the soil cylinder will gain most of the momentum from the buried explosive, which can be seen from the velocity contours in the figure. In fact, peridynamics particles at the top of the spherical dome always have the highest velocities, compared to that of the peridynamic particles in the rest of the dome. Thus these peridynamic particles on the dome top move much faster and consequently make the dome growing larger and larger.

From Fig. 9, one can see that the SPH particles gain very large momentums at the first few steps (see Fig. 9 (a)). The shock waves propagate through the interphase zone (see Fig. $9(\mathrm{~b})$ ), and continuously expand in each direction. After the first wave hits the top of the soil cylinder, the spherical dome is gradually formed by the peridynamic particles at the top.

A full blown-out soil fragmentation profile is displayed in Fig. 10. From Fig. 10, one 


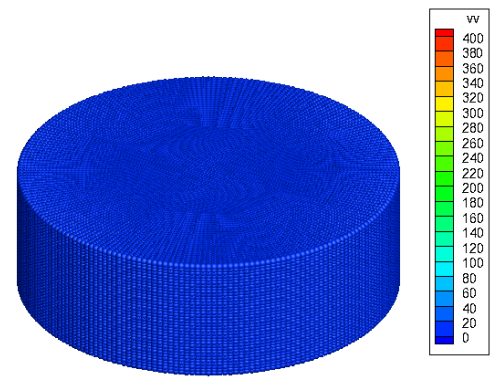

(a) $t=2.45 \mu \mathrm{s}$.

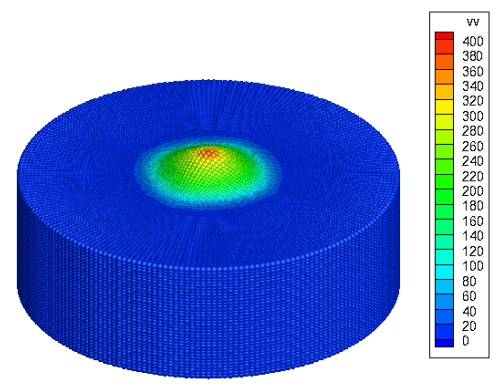

(c) $t=24.5 \mu \mathrm{s}$.

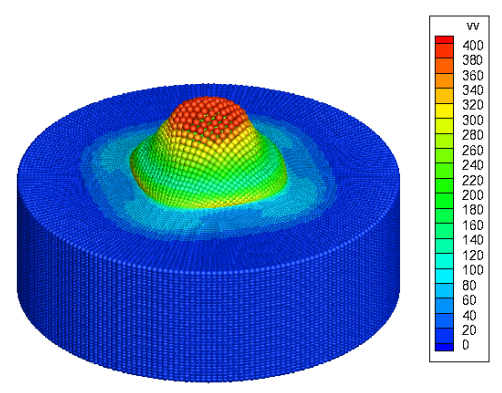

(e) $t=49.00 \mu \mathrm{s}$.

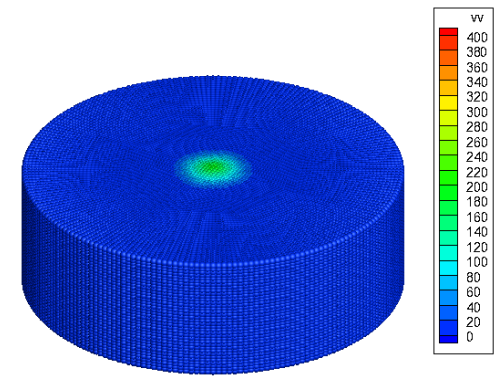

(b) $t=12.25 \mu \mathrm{s}$.

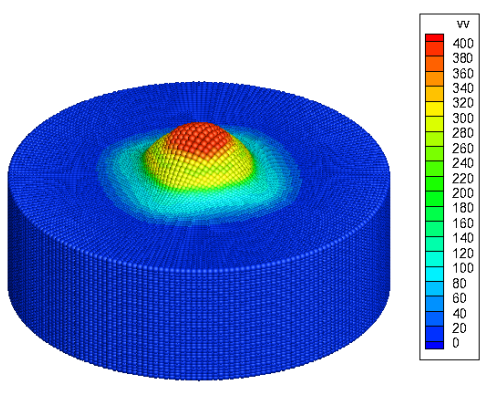

(d) $t=36.75 \mu \mathrm{s}$.

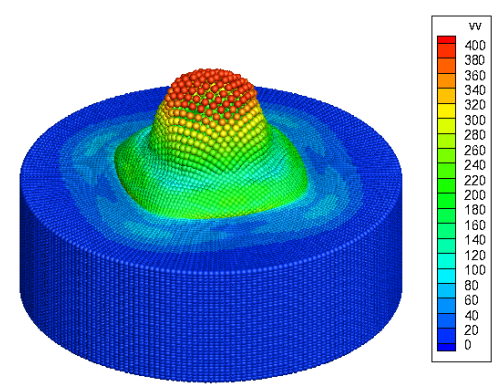

(f) $t=61.25 \mu \mathrm{s}$.

Figure 8: Dynamic fragmentation/ejection process of soil cylinder; view from the top. Contour color: velocity magnitude 


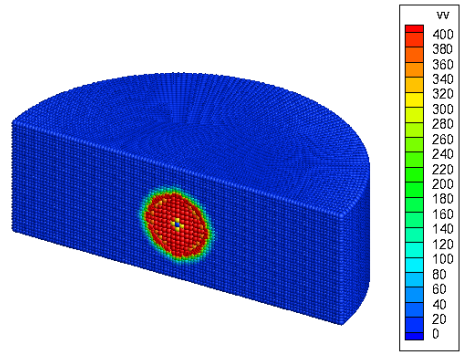

(a) $t=2.45 \mu \mathrm{s}$.

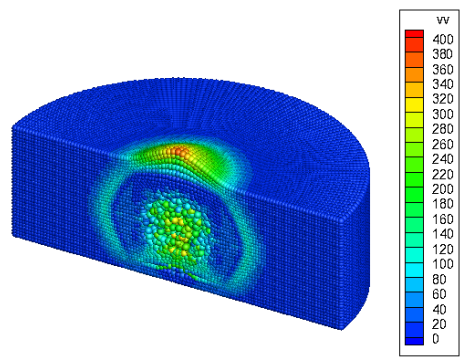

(c) $t=24.5 \mu \mathrm{s}$.

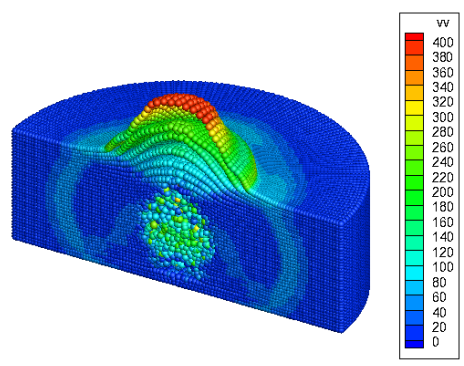

(e) $t=49.00 \mu \mathrm{s}$.

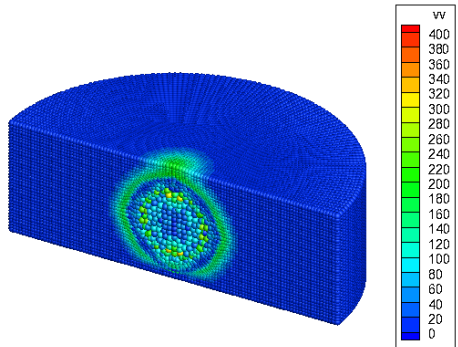

(b) $t=12.25 \mu \mathrm{s}$.

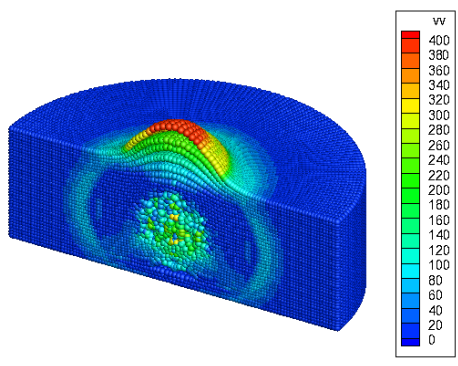

(d) $t=36.75 \mu \mathrm{s}$.

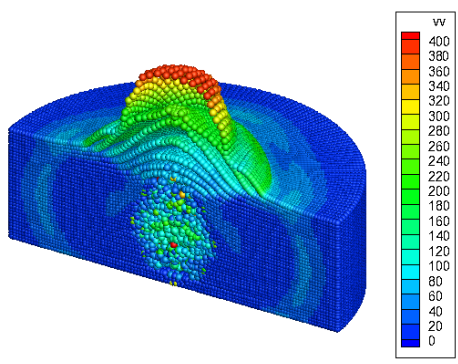

(f) $t=61.25 \mu \mathrm{s}$.

Figure 9: Dynamic fragmentation/ejection process of soil cylinder; view from a vertical cross section. 


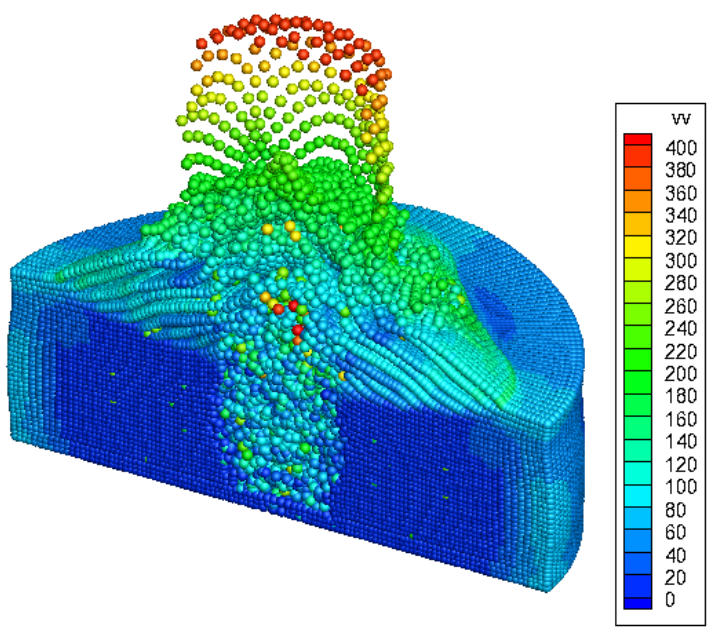

(a) $t=100.00 \mu \mathrm{s}$.

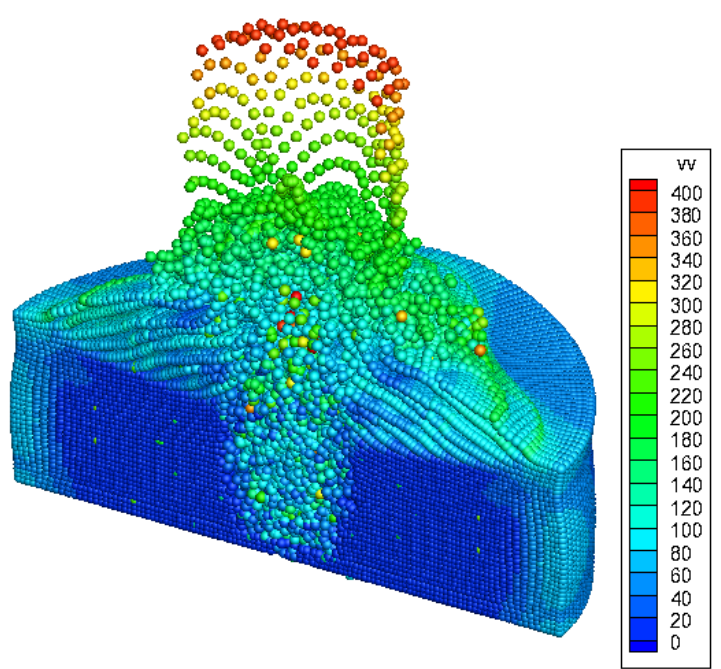

(b) $t=120.0 \mu \mathrm{s}$.

Figure 10: Detailed view of dynamic fragmentation/ejection process of soil cylinder

can measure the height of soil ejection profile and relate that to the explosive strength in experiment data. 


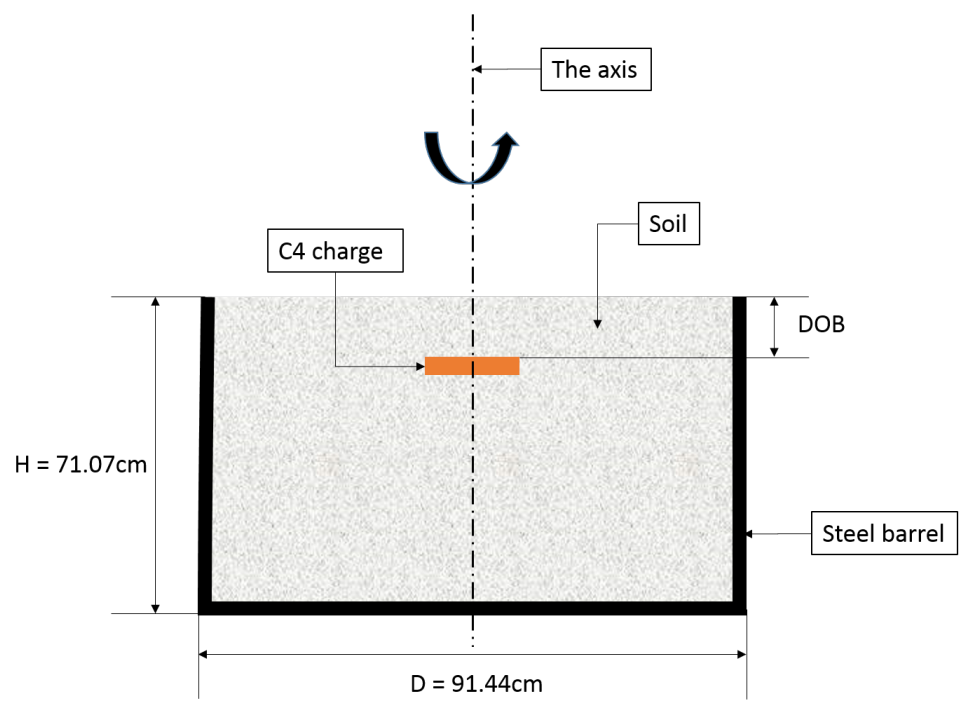

Figure 11: A schematic of the experiment setup used in [28].

To check the validity of this PD-SPH coupling in the simulation of buried explosion, a separate simulation is carried out, in order to compare with the experiment conducted by Bergeron et al. (1998) [28]. Fig. 11] shows the schematic of the experiment setup. A cylindrical barrel with the radius of $45.72 \mathrm{~cm}$ and the height of $71.07 \mathrm{~cm}$ is fully filled with soil. A 100g cylindrical-disk shape C4 explosive charge is buried into the soil along the axis of the barrel with its faces parallel with the soil surface. The distance between the top face of the explosive and the soil surface is defined as the depth of burial (DOB). Two different overburdens with DOBs $3 \mathrm{~cm}$ and $8 \mathrm{~cm}$ are considered in the following simulations. The whole domain is discretized into approximately 1 million particles (generated from a FEM mesh of 949,437 nodes and 928,464 elements), of which 949,132 peridynamic particles are 305 are sph ones. Instead of using the ideal-gas gamma-law relation $p=(\gamma-1) \rho e$, the Jones-Wilkins-Lee (JWL) equation of state is used for the C4 explosive charge, defined as:

$$
p=A\left(1-\frac{\omega}{R_{1} d F}\right) e^{-R_{1} d F}+B\left(1-\frac{\omega}{R_{2} d F}\right) e^{-R_{2} d F}+\frac{\omega e}{V}
$$




\begin{tabular}{|c|c|c|c|c|c|c|}
\hline$\rho$ & $\mathrm{A}$ & $\mathrm{B}$ & $\mathrm{R} 1$ & $\mathrm{R} 2$ & $\omega$ & $\mathrm{E}$ \\
\hline 1601 & $5.98 \times 10^{11}$ & $1.38 \times 10^{9}$ & 4.5 & 1.5 & 0.32 & $8.70 \times 10^{9}$ \\
\hline
\end{tabular}

Table 3: EOS material parameters of the C4 charge ( $m-k g$-s units)

where $p$ is the pressure, $V$ is the volume, $w$ is the Gruneisen parameter, $d F$ is the volume ratio $\frac{V}{V_{0}}$ ( $V_{0}$ is the initial volume) and $A, R_{1}, B, R_{2}, \omega$ (given in Table 3 ) are constants of the C4 charge. The material parameters for the soil and C4 charge are taken from [29]. The simulation time step is chosen to be $\Delta t=0.25 \times 10^{-6} \mathrm{~s}$, with the total time $t=0.8 \times 10^{-3} \mathrm{~s}$. A parallel PD-SPH code is developed by using the OpenMP (an api for shared-memory parallel programming). The simulation takes around 20 hours in an Intel core i7-4700HQ processor with 8 threads involved in the computation. The early soil deformations (soil bubble shapes at the ejecta front) obtained from the simulations are compared with that from the experiments. The time development of the soil bubble height at the ejecta front for both the $3 \mathrm{~cm}$ and $8 \mathrm{~cm}$ overburdens are plotted in Fig. 12. One can see that the simulation results are in general agreement with that of the experiment, which validates the proposed peridynamics soil model to certain extent.

In Fig. 13, we show the simulated soil explosion profile that is in obtained in the numerical simulation of the real experimental results (see Fig. 11), in which one million peridynamaics particles are used.

\section{CONCLUSIONS}

Numerical simulations of fragmentation generated by shock waves from blast loading of the buried explosive has been an outstanding challenge in modeling and simulation. In this work, a hybrid PD-SPH approach is employed to simulate soil fragmentation/ejection. In the coupling PD-SPH framework, a standard Monagphan type artificial viscosity is 


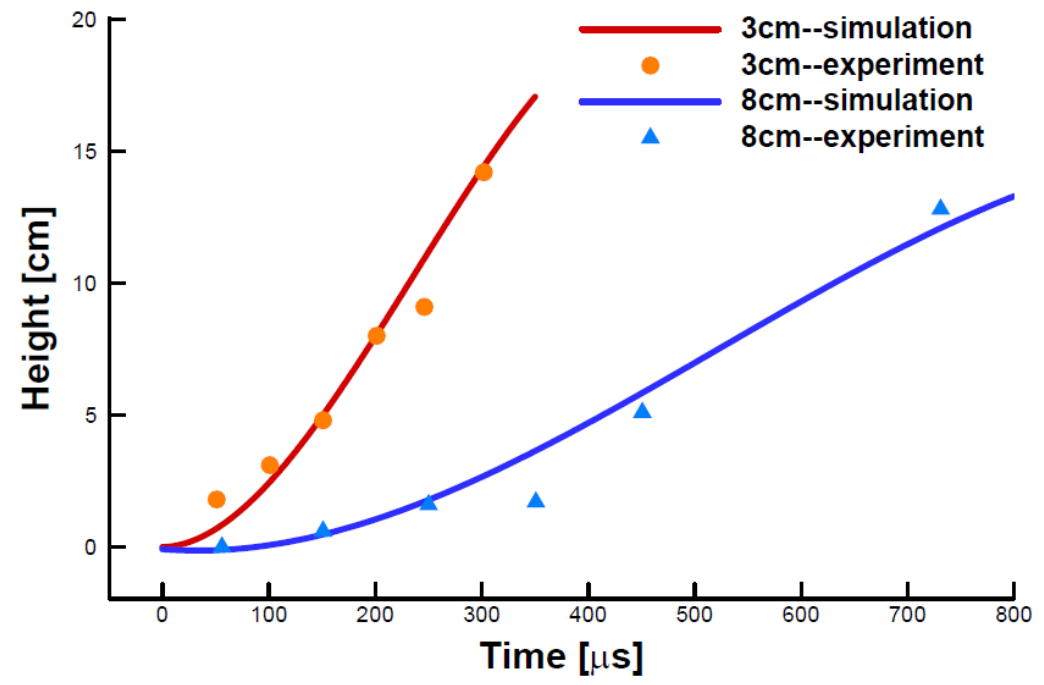

Figure 12: Comparison of the soil bubble heights at the ejecta front with experiment results, for both $3 \mathrm{~cm}$ and $8 \mathrm{~cm}$ overburdens. The height is measured from the top of the soil surface. The experiment data is taken from [28].

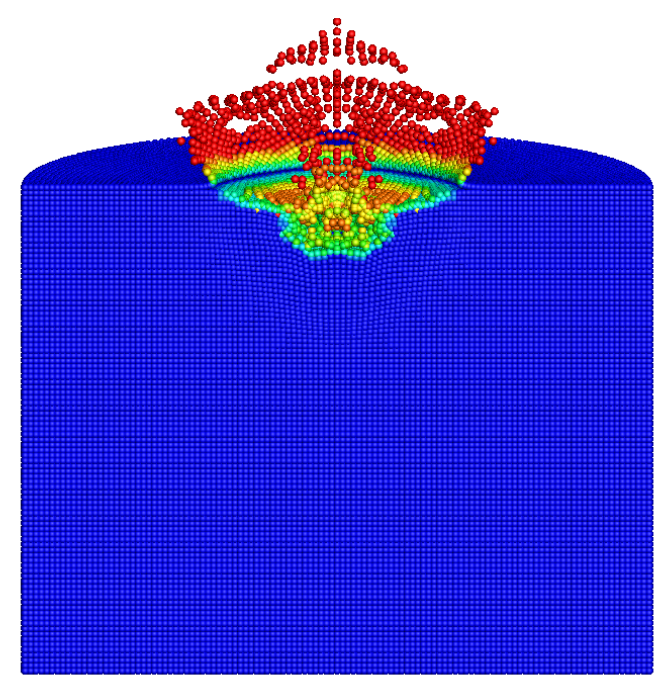

Figure 13: Soil explosion under blast load. 
incorporated into the peridynamics formulation to help in preventing nonphysical particle interpenetrations. For a consistent force and deformation transmissions, by assuming the equivalence of PD-SPH formulations in the current configuration, we have proposed a coupling algorithm of PD-SPH. A Drucker-Prager plasticity soil model is presented at finite strain, with the Hughes-Winget algorithm rephrased in state-based peridynamics framework for the constitutive update.

From the three numerical examples presented, we have found that (1) The Drucker-Prager soil model and its implementations in the state-based peridynamics theory are successful; (2) The proposed PD-SPH coupling scheme works, and (3) the PD-SPH coupling scheme can be used to capture soil fragmentation/ejection induced by the shock waves from blastic loads.

\section{Acknowledgements}

This work was supported by an ONR MURI grant N00014-11-1-0691. This support is gratefully appreciated. 
REFERENCES

[1] S. A. Silling, M. Epton, O. Weckner, J. Xu, and E. Askari. Peridynamic states and constitutive modeling. Journal of Elasticity, 88:151-184, 2007.

[2] J. M. Haile. Molecular dynamics simulation. Wiely, New York, 1992.

[3] T. L. Warren, S. A. Silling, A. Askari, O. Weckner, M. A. Epton, and Jifeng Xu. A non-ordinary state-based peridynamic method to model solid material deformation and fracture. International Journal of Solids and Structures, 46:1186-1195, 2009.

[4] J. T. Foster, S. A. Silling, and W. W. Chen. Viscoplasticity using peridynamics. International Journal for Numerical Methods in Engineering, 81(August 2009):1242-1258, 2010.

[5] O. Weckner and N. Nik Mohamed. Viscoelastic material models in peridynamics. Applied Mathematics and Computation, 219(11):6039-6043, February 2013.

[6] B. K. Tuniki. Peridynamic constitutive model for concrete. PhD thesis, Osmania, 2012.

[7] X. Lai, B. Ren, H. Fan, S. Li, C.T. Wu, A. R. Richard, and L. Liu. Peridynamics simulations of geomaterial fragmentation by impulse loads. International Journal for Numerical and Analytical Methods in Geomechanics, 39:189-213, 2015.

[8] A. Alia and M. Souli. High explosive simulation using multi-material formulations. Applied Thermal Engineering, 26(10):1032-1042, July 2006.

[9] M. B. Liu, G. R. Liu, K. Y. Lam, and Z. Zong. Smoothed particle hydrodynamics for numerical simulation of underwater explosion. Computational Mechanics, 30(2):106118, January 2003. 
[10] R. A. Gingold and J. J. Monaghan. Smoothed particle hydrodynamics—theory and application to non-spherical stars. Monthly notices of the royal astronomical society, 181(3):375-389, 1977.

[11] G. R. Liu and M. B. Liu. Smoothed particle hydrodynamics: a meshfree particle method. World Scientific, 2003.

[12] P. A. Vermeer and R. De Borst. Non-associated plasticity for soils, concrete and rock, 1984.

[13] D.C. Drucker and W. Prager. Soil mechanics and plastic analysis or limit design. Quarterly of Applied Mathematics, 10:157-165, 1952.

[14] H. Matsuoka and T. Nakai. Stress-deformation and strength characteristics of soil under three different principal stresses. Proc. JSCE, 232:59-70, 1974.

[15] L. H. Han, J. A. Elliott, A. C. Bentham, A. Mills, G. E. Amidon, and B. C. Hancock. A modified Drucker-Prager Cap model for die compaction simulation of pharmaceutical powders. International Journal of Solids and Structures, 45:3088-3106, 2008.

[16] L. Q. Torres. Assessment of the applicability of nonlinear Drucker-Prager model with cap to adobe. 15th world conference on earthquake engineering (WCEE), 2012.

[17] B. Ren, H. Fan, G. L. Bergel, R. A. Regueiro, X. Lai, and S. Li. A peridynamicsSPH coupling approach to simulate soil fragmentation induced by shock waves. Computational Mechanics, 55:287-302, 2015.

[18] E. Madenci and E. Oterkus. Peridynamic Theory and Its Applications. Springer, Berlin, 2014. 
[19] S. A. Silling and E. Askari. A meshfree method based on the peridynamic model of solid mechanics. Computers \& Structures, 83(17-18):1526-1535, June 2005.

[20] M. R. Tupek and R. Radovitzky. An extended constitutive correspondence formulation of peridynamics based on nonlinear bond-strain measures. Journal of the Mechanics and Physics of Solids, 65:82-92, April 2014.

[21] D. A. Fulk. A Numerical Analysis of Smoothed Particle Hydrodynamics. PhD thesis, Air Force Institute of Technology, 1994.

[22] T. Rabczuk, T. Belytschko, and S. P. Xiao. Stable particle methods based on Lagrangian kernels. Computer Methods in Applied Mechanics and Engineering, 193(12-14):1035-1063, March 2004.

[23] J. J. Monagphan. Simulating free surface flows with SPH. Journal of Computational Physics, 110(2):339-406, 1994.

[24] O. C. Zienkiewicz, A. H. C. Chan, M. Pastor, B. A. Schrefler, and T. Shiomi. Computational Geomechanics. Wiley Chichester, 1999.

[25] T. Belytschko, W. K. Liu, and B. Moran. Nonlinear Finite Elements For Continua And Structures, volume 104. 2000.

[26] T. J. R. Hughes and J. Winget. Finite rotation effects in numerical integration of rate constitutive equations arising in large-deformation analysis. International Journal for Numerical Methods in Engineering, 15:1862-1867, 1980.

[27] B. Kilic and E. Madenci. An adaptive dynamic relaxation method for quasi-static simulations using the peridynamic theory. Theoretical and Applied Fracture Mechanics, 53(3):194-204, June 2010. 
[28] D. Bergeron, R. Walker, and C. Coffey. Detonation of 100-gram anti-personnel mine surrogate charges in sand-a test case for computer code validation. Technical report, Defence Research Establishment Suffield, Ralston ALTA (CAN), 1998.

[29] D. Dooge, R. Dwarampudi, G. Schaffner, and A. Miller. Evolution of occupant survivability simulation framwork using FEM-SPH coupling. In 2011 NDIA ground vehicle systems engineering and technology symposium, Dearborn, Michigan, 2011. 